\title{
An Alternate Generalized Odd Generalized Exponential Family with Applications to Premium Data
}

\author{
Sadaf Khan ${ }^{1, * \mathbb{D}}$, Oluwafemi Samson Balogun ${ }^{2, * \mathbb{D}}$, Muhammad Hussain Tahir ${ }^{1} \mathbb{D}$, Waleed Almutiry ${ }^{3} \mathbb{D}$ \\ and Amani Abdullah Alahmadi 4 (D)
}

1 Department of Statistics, The Islamia University of Bahawalpur, Bahawalpur 63100, Pakistan; mht@iub.edu.pk

2 School of Computing, University of Eastern Finland, 70211 Kuopio, Northern Europe, Finland

3 Department of Mathematics, College of Science and Arts in Ar Rass, Qassim University, Buryadah 52571, Saudi Arabia; wkmtierie@qu.edu.sa

4 College of Science and Humanities, Shaqra University, Shaqra 15572, Saudi Arabia; aalahmadi@su.edu.sa

* Correspondence: drsmkhan22@gmail.com (S.K.); samson.balogun@uef.fi (O.S.B.)

check for updates

Citation: Khan, S.; Balogun, O.S.; Tahir, M.H.; Almutiry, W.; Alahmadi, A.A. An Alternate Generalized Odd Generalized Exponential Family with Applications to Premium Data. Symmetry 2021, 13, 2064. https:// doi.org/10.3390/sym13112064

Academic Editor: Zhivorad Tomovski

Received: 19 September 2021

Accepted: 14 October 2021

Published: 1 November 2021

Publisher's Note: MDPI stays neutral with regard to jurisdictional claims in published maps and institutional affiliations.

Copyright: (c) 2021 by the authors. Licensee MDPI, Basel, Switzerland. This article is an open access article distributed under the terms and conditions of the Creative Commons Attribution (CC BY) license (https:/ / creativecommons.org/licenses/by/ $4.0 /)$.

\begin{abstract}
In this article, we use Lehmann alternative-II to extend the odd generalized exponential family. The uniqueness of this family lies in the fact that this transformation has resulted in a multitude of inverted distribution families with important applications in actuarial field. We can characterize the density of the new family as a linear combination of generalised exponential distributions, which is useful for studying some of the family's properties. Among the structural characteristics of this family that are being identified are explicit expressions for numerous types of moments, the quantile function, stress-strength reliability, generating function, Rényi entropy, stochastic ordering, and order statistics. The maximum likelihood methodology is often used to compute the new family's parameters. To confirm that our results are converging with reduced mean square error and biases, we perform a simulation analysis of one of the special model, namely OGE2-Fréchet. Furthermore, its application using two actuarial data sets is achieved, favoring its superiority over other competitive models, especially in risk theory.
\end{abstract}

Keywords: generalized exponential distribution; generalized exponential distribution; OGE-G family; Rényi entropy; order statistic

\section{Introduction}

In recent years, there has been a dramatic growth in the number of generalisations of well-known probability distributions. Most notable generalizations are achieved by (i) inducting power parameters in well established parent distributions, (ii) extending the classical distribution by modification in their functions, (iii) introducing special functions such as $\mathrm{W}[\mathrm{K}(\mathrm{x})]$ as generators and (iv) by compounding of distributions. This heaped surge of generalized families is due to the flexibility in modelling phenomenons related to the changing scenarios of contemporary scientific field including demography, actuarial, survival, biological, ecological, communication theory, epidemiology and environmental sciences. However, a clear understanding of the applicability of these models in most applied areas is necessary if one is to gain insights into systems that can be modeled as random processes. The model, thus obtained, acquires improved empirical results to the real data that is collected adaptively.

Although there exist many functions which act as generators to produce flexible classes of distributions, in this project, we will emphasize generalizations in which a ratio of survival function (sf) has been used in some form, commonly known as the odd ratio. In the reference [1], a proportional odd family viz. a viz. the Marshall Olkin-G (MO-G) was generalized by sf $\bar{K}(x)=1-K(x)$, where $K(x)$ is the distribution function (cdf) of parent distribution, with the induction of a tilt parameter. Gleaton and Lynch, in the reference [2], 
used the odd function as generator when they defined a log odd family (OLL-G). In the reference [3], defined the odd Weibull family, as an asymptotically equivalent log-logistic model for larger values of $\theta$, the scale parameter. The reference [4] used the Transformed -Transformer (TX) family, due to the reference [5], to define odd Weibul-G families of distribution. Since then, a myriad of distributions has been generalized using odd function. Some of the important families include [6-31], among others.

Focussing on the origins and motivations of our proposed scheme, the authors in [32] proposed the odd generalized exponential family (which we refer to OGE-G) as a better alternative to generalized exponential (GE) family using Lehmann Alternative-I (LA-I). The cdf of the two parameter OGE-G family is mentioned below:

$$
F_{O G E-G}(x ; \alpha, \lambda, \psi)=\left(1-\mathrm{e}^{-\lambda \frac{K(x ; \psi)}{\bar{K}(x ; \psi)}}\right)^{\alpha}, \quad x>0, \alpha>0, \lambda>0 .
$$

In the reference [33], an odd family of GE was proposed so-called generalized odd generalized exponential family (which we refer to OGE1-G). The cdf of OGE1-G family is presented as:

$$
F_{O G E 1-G}(x ; \alpha, \beta, \psi)=\left(1-\mathrm{e}^{-\frac{K^{\alpha}(x ; \psi)}{1-K^{\alpha}(x ; \psi)}}\right)^{\beta}, \quad x>0, \alpha>0, \beta>0 .
$$

Because of its capacity to simulate variable hazard rate function (hrf) forms of all traditional types in lifetime data analysis, we believe OGE-G offers a sensible combination of simplicity and flexibility. However, the relevance of OGE1-G to lifespan modelling in domains such as reliability, actuarial sciences, informatics, telecommunications, and computational social sciences (just to highlight a few) is still debatable. According to the reference [34], the Lehmann Alternative-II (LA2) approach has received less attention. This motivated us to use LA2 approach to develop the exponentiated odd generalized exponential (OGE2-G), in the same vein as OGE-G and OGE1-G. Adhering to the framework defined in the reference [5], if $T$ follows GE random variable (rv), then the cdf of OGE2-G family is mentioned below:

$$
F_{O G E 2-G}(x ; \alpha, \beta, \psi)=\left[1-\mathrm{e}^{-\frac{1-\bar{K}(x ; \psi)^{\alpha}}{\bar{K}(x ; \psi)^{\alpha}}}\right]^{\beta}, \quad x>0, \alpha, \beta>0
$$

where $\alpha$ and $\beta$ are shape parameter and $\psi$ is the vector of baseline parameter.

Consider the following points to emphasise the model's distinctiveness; (i) In the literature, the proposed model in its current form has not been studied to the best of our knowledge, (ii) From an analytical standpoint, the OGE2-G family has a significantly better configuration and practicality than OGE-G and OGE1-G for inverted models with minimal chance to counter non-identifiability issues, (iii) The OGE2-G has several curious connections to other families. When $\alpha$ approaches $0, F(x ; \alpha, \beta, \psi)$ tends to GE with $\lambda=1$, when $\alpha=1 F(x ; \alpha, \beta, \psi)$ tends to OGE-G, if $\alpha \rightarrow 0$ and $\beta=1$ then $F(x ; \alpha, \beta, \psi)$ tends to odd exponential (OE) (iv) This new dimension allowed us to explore models which are naturally constituted by LA2. The generalizations, thus attained, produced skewed distributions with much heavier tails enabling its practicality in risk evaluation theory with far better results, (v) The successful application of OGE2-G family motivates future research, as it outperforms nine well-established existing models, (vi) We present a physical explanation for $X$ when $\alpha$ and $\beta$ are integers. Consider there be a parallel system consisting of $\beta$ identically independent components. Suppose that the lifetime of a rv $Y$ with a specific $K(x ; \psi)$ with $\alpha$ components in a series system such that the risk of failing at time $x$ is 
represented by the odd function as $\frac{1-\bar{K}(x ; \psi)^{\alpha}}{\bar{K}(x ; \psi)^{\alpha}}$. Consider that the randomness of this risk is represented by the $\mathrm{rv} X$, then we can assume the following relation holds

$$
\operatorname{Pr}(Y \leq x)=\operatorname{Pr}\left(X \leq \frac{1-\bar{K}(x ; \psi)^{\alpha}}{\bar{K}(x ; \psi)^{\alpha}}\right)=F(x ; \alpha, \beta, \psi),
$$

explicitly given in Equation (1). The OGE2-G family is offered and explored in this research, emphasising its diversity and scope for application to real life phenomenons. The major features of the OGE2-G family, including the pdf, hrf, qf, and ten unique models from OGE2-G family presented in Table 1, are provided in the first half. Then, certain mathematical properties of the OGE2-G such as series expansion of the exponentiated pdf, moments, parameter estimation, order statistics, Rényi entropy, stress-strength analysis and stochastic dominance results are investigated. Furthermore, Fréchet is specified as baseline model termed as OGE2-Fréchet (denoted as OGE2Fr) and the maximum likelihood (ML) technique is then used to construct statistical applications of the special model. We choose to study OGE2Fr specifically as its nested model include inverse-Rayleigh (IR) and inverse exponential (IE), favoring its suitability over sub-models as well. It is applied to fit two sets of premium data from actuarial field. Using key performance indicators, we reveal that OGE2Fr outperforms nine competing models. A portion pertinent to specific risk measures, with an emphasis on the value at risk (VaR) and the expected shortfall (ES), is presented. Eventually, the estimation of risk measures for the examined data sets is then discussed, with the proposed methodology yielding a rather satisfying result. Equation (1) can be useful in modelling real life survival data with different shapes of hrf. Table 1 lists $\frac{1-\bar{K}(x ; \psi)^{\alpha}}{\bar{K}(x ; \psi)^{\alpha}}$ and the corresponding parameters for some special distributions which are considered to be the potential sub-models of OGE2-G family.

Table 1. Distributions and corresponding $\frac{1-\bar{K}(x ; \psi)^{\alpha}}{\bar{K}(x ; \psi)^{\alpha}}$ functions.

\begin{tabular}{ccc}
\hline Distribution & $\frac{1-\bar{K}(x ; \psi)^{\alpha}}{\overline{\mathbf{K}}(x ; \psi)^{\alpha}}$ & $\psi$ \\
\hline Fréchet $(x>0)$ & {$\left[1-\mathrm{e}^{(a / x)^{b}}\right]^{-\alpha}-1$} & $(a, b)$ \\
Generalized exponential $(x>0)$ & {$\left[1-\left(1-\mathrm{e}^{-\lambda x}\right)^{\theta}\right]^{-\alpha}-1$} & $(\lambda, \theta)$ \\
Power function $(0<x<a)$ & {$\left[1-(x / a)^{b}\right]^{-\alpha}-1$} & $(a, b)$ \\
Burr III $(x>0)$ & {$\left[1-\left(1+x^{-c}\right)^{-k}\right]^{-\alpha}-1$} & $(c, k)$ \\
Half-logistic $(x>0)$ & {$\left[2\left(\mathrm{e}^{x}+1\right)^{-1}\right]^{-\alpha}-1$} & $(\alpha)$ \\
Log-logistic $(x>0)$ & {$\left[1+(x / a)^{b}\right]^{\alpha}-1$} & $(a, b)$ \\
Inverse Rayleigh $(x>0)$ & {$\left[1-\mathrm{e}^{\gamma / x^{2}}\right]^{-\alpha}-1$} & $(\gamma)$ \\
Inverse Exponential $(x>0)$ & {$\left[1-\mathrm{e}^{\gamma / x}\right]^{-\alpha}-1$} & $(\gamma)$ \\
Normal $(-\infty<x<\infty)$ & {$\left[1-\phi\left(\frac{x-\mu}{\sigma}\right)\right]^{-\alpha}-1$} & $(\mu, \sigma)$ \\
Gumbel $(-\infty<x<\infty)$ & {$\left[1-\mathrm{e}^{\left.-\mathrm{e}^{\frac{x-\mu}{\sigma}}\right]^{-\alpha}-1}\right.$} & {$[\mu, \sigma)$} \\
\hline
\end{tabular}

The following is a breakdown of how the paper is constructed. In Section 2, we acquaint the readers to the new family with basic properties and ten potential baseline models which can become members of OGE2-G family. Section 3 is comprised of the mathematical properties of the OGE2-G family. Section 4 progresses by taking Fréchet (Fr) as sub-model to propose OGE2Fr and related statistical and inferential properties. Section 5 specifies two applications of actuarial data sets with emphasis on risk evaluation (premium returns) and the proposed model's veracity is established. Furthermore, the 
model is applied to compute some actuarial measures. Section 6 is the final section, with some annotations and useful insights.

\section{The OGE2G Family}

In this segment, basic statistical properties of the newly proposed family characterized by the cdf, in Equation (1) are presented. Functional forms of ten sub models are also defined.

\subsection{Definition of pdf and hrf}

The pdf in agreement with Equation (1) is given as (2).

$$
f_{O G E 2-G}(x ; \alpha, \beta, \psi)=\alpha \beta k(x ; \psi) \bar{K}(x ; \psi)^{-\alpha-1} \mathrm{e}^{-\left\{\frac{1-\bar{K}(x ; \psi)^{\alpha}}{\bar{K}(x ; \psi)^{\alpha}}\right\}}\left[1-\mathrm{e}^{-\left\{\frac{1-\bar{K}(x ; \psi)^{\alpha}}{\bar{K}(x ; \psi)^{\alpha}}\right\}}\right]^{\beta-1}
$$

Using the results defined in Equations (1) and (2), the hrf is defined as

$$
\begin{aligned}
\tau(x ; \alpha, \beta, \psi)= & \frac{f_{\text {OGE2-G }}(x ; \alpha, \beta, \psi)}{1-F_{\text {OGE2 }-G}(x ; \alpha, \beta, \psi)} \\
= & \alpha \beta k(x ; \psi) \bar{K}(x ; \psi)^{-\alpha-1} \mathrm{e}^{-\left\{\frac{1-\bar{K}(x ; \psi)^{\alpha}}{\bar{K}(x ; \psi)^{\alpha}}\right\}}\left[1-\mathrm{e}^{-\left\{\frac{1-\bar{K}(x ; \psi)^{\alpha}}{\bar{K}(x ; \psi)^{\alpha}}\right\}}\right]^{\beta-1} \\
& \times\left[1-\left(1-\mathrm{e}^{-\left\{\frac{1-\bar{K}(x ; \psi)^{\alpha}}{\bar{K}(x ; \psi)^{\alpha}}\right\}}\right)^{\beta-1}\right]
\end{aligned}
$$

The hazard rate is just a calculation of the change in survivor rate per unit of time. Hence, its importance in reliability and survival analysis is crucial. The hrf has some characteristic shapes which include monotonic (increasing, decreasing), non-monotonic (bathtub or upside down bathtub) or constant. Standard statistical distribution yield maximum three shapes, but OGE2-G family can yield a diverse range of shapes (including increasing-decreasing-increasing) depending upon the choice of special model. For further details on hrf, see [35].

\subsection{Quantile Function and Potential Sub-Models}

The OGE2-G family may be readily approximated by reversing Equation (1) as shown below: If indeed the distribution of $u$ is uniform $u(0,1)$, therefore

$$
x=Q_{K}\left[1-\left\{1-\log \left(1-u^{1 / \beta}\right)\right\}^{-1 / \alpha}\right] .
$$

Equation (4) can be useful to define statistical measures such as median, skewness, and kurtosis based on quartiles, deciles, or percentiles. These measures facilitates to concisely define the skewness and kurtosis measures which are significant tool to comprehend the shape(s) of the distribution.

Theorem 1 shows how the OGE2 family is related to other distributions.

Theorem 1. Let $X \sim O G E 2-G(\alpha, \beta ; \psi)$, then

(a) If $Y=1-\bar{K}(x ; \psi)^{\alpha}$, then $F_{Y}(y)=\left(1-\mathrm{e}^{-\frac{Y}{1-Y}}\right)^{\beta}, \quad 0<y<1$, and

(b) If $Y=\frac{1-\bar{K}(x ; \psi)^{\alpha}}{\bar{K}(x ; \psi)^{\alpha}}$, then $Y \sim G E(1, \beta)$. 


\section{Mathematical Properties of OGE2-G Family}

To capture the family's modelling capacity, numerous mathematical features of the OGE2-G are examined in this section. Some of the key results established in this section are then applied in Section 5.

\subsection{Linear Expansion of $c d f$}

We provide a useful expansion for (1) in terms of linear combinations of exp-G density functions using the following series expansion as

$$
(1-z)^{\eta-1}=\sum_{i=0}^{\infty} \frac{(-1)^{i} \Gamma(\eta)}{i ! \Gamma(\eta-i)} z^{i}
$$

whereas the expansion holds for all $|z|<1$ and $\eta>0$ a non-integer value. Then, the cdf of OGE2-G class in (1) can indeed be phrased with

$$
F(x)=\sum_{i=0}^{\infty} \frac{(-1)^{i} \Gamma(\beta+1)}{i ! \Gamma(\beta+1-i)} \mathrm{e}^{-i\left(\frac{1-\overline{\mathrm{K}}^{\mathrm{ff}}}{\overline{\mathrm{K}}^{\mathrm{ff}}}\right)} .
$$

Using series expansion and power series expansion in Equation (5), will yield the following cdf

$$
F(x)=F(x: \alpha, \beta, \psi)=\sum_{\ell=0}^{\infty} \xi_{\ell} H_{\ell}(x),
$$

where $H_{\ell}(x)=K(x ; \psi)^{\ell}$ (for $\ell \geq 1$ ) denotes the cdf of exp-G distribution with power parameter $\ell$ and

$$
\xi_{\ell}=(-1)^{\ell} \sum_{i, j=1}^{\infty} \sum_{k=0}^{j}(-1)^{i+j+k} \frac{\Gamma(\beta+1)}{i ! j ! \Gamma(\beta+1-i)}\left(\begin{array}{l}
j \\
k
\end{array}\right)\left(\begin{array}{c}
\alpha(k-i) \\
\ell
\end{array}\right) .
$$

Through differentiating Equation (6) the OGE2-G family density, we may express it as a combination of exp-G densities.

$$
f(x: \alpha, \beta, \psi)=\sum_{i, j=1}^{\infty} \xi_{\ell} h_{\ell}(x)
$$

where $h_{\ell}(x)=\ell K^{\ell-1}(x ; \psi) k(x ; \psi)$ is the exp-G pdf with power parameter $\ell$. As a result, numerous features of the proposed model may be deduced from the exp-G distribution's attributes. Most modern computation frameworks, such as MathCad, Maple, Mathematica, and Matlab, can efficiently handle the formulas derived throughout the article, which can currently operate using the use of analytic formulations of enormous size and complexity.

\subsection{Numerous Types of Moments}

The fundamental formula for the $p$ th moment of $X$ is supplied by (7) as

$$
\mu_{p}^{\prime}=\sum_{i, j=0}^{\infty} \xi_{\ell} E\left(X_{\ell}^{p}\right)
$$

where $\mathbb{E}\left(X_{\ell}^{p}\right)=\int_{0}^{\infty} x^{p} h_{\ell}(x) d x$. Setting $p=1$ in (8) can provide explicit expression for the mean of several parent distributions. 
A another expression for $\mu_{p}^{\prime}$ is taken from (8) as far as the baseline qf is concerned

$$
\mu_{p}^{\prime}=\sum_{i, j=0}^{\infty} \xi_{\ell} \tau(p, \ell-1) .
$$

where $\tau(p, \ell-1)=\int_{0}^{1} Q_{G}(u)^{p} u^{\ell} d u$.

The central moments $\left(\mu_{p}\right)$ and cumulants $\left(\kappa_{p}\right)$ of $X$ can follow from Equation (8) as $\mu_{p}=\sum_{k=0}^{p}\left(\begin{array}{c}p \\ k\end{array}\right)(-1)^{k} \mu_{1}^{\prime p} \mu_{p-k}^{\prime}$ and $\kappa_{s}=\mu_{s}^{\prime}-\sum_{k=1}^{s-1}\left(\begin{array}{c}s-1 \\ k-1\end{array}\right) \kappa_{k} \mu_{s-k^{\prime}}^{\prime}$ respectively, where $\kappa_{1}=\mu_{1}^{\prime}$.

The $r$ th lower incomplete moment of $X$ can be determined from Equation (7) as

$$
m_{r}(y)=\sum_{i, j=0}^{\infty} \xi_{\ell} \int_{0}^{G(y)} Q_{G}(u)^{r} u^{l-1} d_{u}
$$

For most $G$ distributions, the final integral may be calculated.

\subsection{Inference Related to OGE2 Family}

The strategy of maximum likelihood (MLL) approach is used to estimate the unknown parameters of the new class. Let $x_{1}, \ldots, x_{n}$ be $n$ observations from the OGE2-G density class (2) with parameter vector $\Theta=(\alpha, \beta, \psi)^{\top}$. Then the likelihood function $\mathcal{L}(\alpha, \beta, \delta)$ on the domain $\Theta$ is defined as

$$
\begin{aligned}
\mathcal{L}= & n \log (\alpha)+n \log (\beta)+\sum_{i=1}^{n} \log k\left(x_{i} ; \psi\right)-(\alpha+1) \log \bar{K}\left(x_{i} ; \psi\right) \\
& -V\left(x_{i} ; \alpha, \psi\right)+(\beta-1) \sum_{i=1}^{n} \log \left[1-\mathrm{e}^{-V\left(x_{i} ; \alpha, \psi\right)}\right],
\end{aligned}
$$

where $V\left(x_{i} ; \alpha, \psi\right)=\frac{1-\bar{K}\left(x_{i} ; \psi\right)^{\alpha}}{\bar{K}\left(x_{i} ; \psi\right)^{\alpha}}$.

The elements of the score vector $U(\Theta)$ are as described in the following:

$$
\begin{aligned}
U_{\alpha}= & \frac{n}{\alpha}-\sum_{i=1}^{n} \log \bar{K}\left(x_{i} ; \psi\right)-V^{\prime(\alpha)}\left(x_{i} ; \alpha, \psi\right) \\
& +\sum_{i=1}^{n} \frac{(\beta-1) \mathrm{e}^{-V\left(x_{i} ; \alpha, \psi\right)} V^{\prime(\alpha)}\left(x_{i} ; \alpha, \psi\right)}{\left(1-\mathrm{e}^{-V\left(x_{i} ; \alpha, \psi\right)}\right)}, \\
U_{\beta}= & \frac{n}{\beta}+\sum_{i=1}^{n} \log \left[1-\mathrm{e}^{-V\left(x_{i} ; \alpha, \psi\right)}\right] \\
U_{\psi}= & \sum_{i=1}^{n}\left[\frac{k^{\prime}\left(x_{i} ; \psi\right)}{k\left(x_{i} ; \psi\right)}\right]+(\alpha+1) \sum_{i=1}^{n}\left[\frac{k\left(x_{i} ; \psi\right) k^{\prime}\left(x_{i} ; \psi\right)}{\bar{K}\left(x_{i} ; \psi\right)}\right]-V^{\prime\left(\xi_{k}\right)}\left(x_{i} ; \alpha, \psi\right) \\
& +(\beta-1) \sum_{i=1}^{n}\left[\frac{\mathrm{e}^{-V\left(x_{i} ; \alpha, \psi\right)}\left\{V^{\prime\left(\xi_{k}\right)}\left(x_{i} ; \alpha, \psi\right)\right\}}{\left.1-\mathrm{e}^{-V\left(x_{i} ; \alpha, \psi\right)}\right]}\right.
\end{aligned}
$$

where $V^{\prime(\alpha)}($.$\left.) and V^{\prime} \psi_{k}\right)($.$) means the derivative of the function V$ with respect to $\alpha$ and $\psi$, respectively.

The next elements are produced by the components of the score vector $J(\Theta)$. 


$$
\begin{aligned}
& J_{\alpha \alpha}=-\frac{n}{\alpha^{2}}+\sum_{i=1}^{n} \frac{k\left(x_{i} ; \alpha, \beta, \psi_{k}\right) V^{\prime\left(\psi_{k}\right)}}{\bar{K}\left(x_{i} ; \alpha, \beta, \psi_{k}\right)}-V^{\prime \prime(\alpha \alpha)}+(\beta-1) \\
& \times \sum_{i=1}^{n} \frac{\mathrm{e}^{-V\left(x_{i} ; \alpha, \psi\right)} V^{\prime}\left(x_{i} ; \alpha, \alpha\right)+\mathrm{e}^{-V\left(x_{i} ; \alpha, \psi\right)}\left(V^{\prime}\left(x_{i} ; \alpha\right)\right)^{2}}{\left(1-\mathrm{e}^{-V\left(x_{i} ; \alpha, \psi\right)}\right)^{2}}, \\
& J_{\alpha \beta}=\sum_{i=1}^{n} \frac{\mathrm{e}^{-V\left(x_{i} ; \alpha, \psi\right)} V^{\prime \alpha}\left(x_{i} ; \alpha, \psi\right)}{\left[1-\mathrm{e}^{-V\left(x_{i} ; \alpha, \psi\right)}\right]^{2}} \text {, } \\
& J_{\alpha \psi}=\sum_{i=1}^{n} \frac{k\left(x_{i} ; \alpha, \beta, \psi\right) k^{\prime}\left(x_{i} ; \alpha, \beta, \psi\right)}{\bar{K}\left(x_{i} ; \alpha, \beta, \psi\right)}-V^{\prime \prime}(\alpha \psi)\left(x_{i} ; \alpha, \psi\right)+(\beta-1) \\
& \times \sum_{i=1}^{n} \frac{\left[\mathrm{e}^{-V\left(x_{i} ; \alpha, \psi\right)}\right]^{2} V^{\prime(\alpha)}\left(x_{i} ; \alpha, \psi\right) V^{\prime(\psi)}\left(x_{i} ; \alpha, \psi\right)-\mathrm{e}^{-V\left(x_{i} ; \alpha, \psi\right)} V^{\prime \prime(\alpha \psi)}\left(x_{i} ; \alpha, \psi\right)}{\left(1-\mathrm{e}^{-V\left(x_{i} ; \alpha, \psi\right)}\right)^{2}} \\
& -(\beta-1) \sum_{i=1}^{n} \frac{\mathrm{e}^{-V\left(x_{i} ; \alpha, \beta, \psi\right)} V^{\prime(\alpha)}\left(x_{i} ; \alpha, \psi\right) V^{\prime(\psi)}\left(x_{i} ; \alpha, \psi\right)}{\left(1-\mathrm{e}^{-V\left(x_{i} ; \alpha, \psi\right)}\right)^{2}} \\
& +(\beta-1) \sum_{i=1}^{n} \frac{\left.\left[\mathrm{e}^{-V\left(x_{i} ; \alpha, \psi\right)}\right]^{2} V^{\prime(\alpha)}\left(x_{i} ; \alpha, \psi\right) V^{\prime(\psi}\right)\left(x_{i} ; \alpha, \psi\right)}{\left(1-\mathrm{e}^{-V\left(x_{i} ; \alpha, \psi\right)}\right)^{2}}, \\
& J_{\beta \beta}=-\frac{n}{\beta^{2}}, \\
& J_{\beta \psi}=\sum_{i=1}^{n} \frac{\mathrm{e}^{-V\left(x_{i} ; \alpha, \psi\right)} V^{\prime(\psi)}\left(x_{i} ; \alpha, \psi\right)}{1-\mathrm{e}^{-V\left(x_{i} ; \alpha, \psi\right)}}, \\
& J_{\psi \psi}=\sum_{i=1}^{n} \frac{k\left(x_{i ; \psi}\right) k^{\prime \prime}\left(x_{i ; \psi}\right)-\left[k^{\prime}\left(x_{i ; \psi}\right)\right]^{2}}{\left[k\left(x_{i ; \psi}\right)\right]^{2}}+(\alpha+1) \sum_{i=1}^{n}\left[\frac{k\left(x_{i ; \psi}\right) k^{\prime}\left(x_{i ; \psi}\right)\left\{-k^{\prime}\left(x_{i ; \psi}\right)\right\}^{2}}{\bar{K}^{2}\left(\left(x_{i ; \psi}\right)\right)}\right] \\
& -V^{\prime \prime}(\psi \psi)\left(x_{i ; \psi}\right)-(\beta-1) \sum_{i=1}^{n} \frac{\left[\mathrm{e}^{-V\left(x_{i} ; \alpha, \psi\right)} V^{\prime(\psi)}\left(x_{i} ; \alpha, \psi\right)\right]^{2}}{\left(1-\mathrm{e}^{-V\left(x_{i} ; \alpha, \psi\right)}\right)^{2}} \\
& -(\beta-1) \sum_{i=1}^{n} \frac{\mathrm{e}^{-V\left(x_{i} ; \alpha, \psi\right)} V^{\prime \prime(\psi \psi)}\left(x_{i} ; \alpha, \psi\right)+\mathrm{e}^{-V\left(x_{i} ; \alpha, \psi\right)}\left[V^{\prime(\psi)}\left(x_{i} ; \alpha, \psi\right)\right]^{2}}{\left(1-\mathrm{e}^{-V\left(x_{i} ; \alpha, \psi\right)}\right)^{2}} \\
& +(\beta-1) \sum_{i=1}^{n} \frac{\left[\mathrm{e}^{-V\left(x_{i} ; \alpha, \psi\right)}\right]^{2} V^{\prime \prime(\psi \psi)}\left(x_{i} ; \alpha, \psi\right)+\left[\mathrm{e}^{-V\left(x_{i} ; \alpha, \psi\right)} V^{\prime(\psi)}\left(x_{i} ; \alpha, \psi\right)\right]^{2}}{\left(1-\mathrm{e}^{-V\left(x_{i} ; \alpha, \psi\right)}\right)^{2}},
\end{aligned}
$$

where $V^{\prime \prime}(\alpha \alpha)($.$) is the derivative of V^{\prime(\alpha)}($.$) with respect to \alpha, V^{\prime \prime}(\alpha \psi)($.$) is the derivative$ of $V^{\prime \alpha}($.$) with respect to \psi_{k}$ and $V^{\prime \prime} \psi \psi($.$) is the derivative of V^{\prime(\psi)}($.$) with respect to \psi$.

\subsection{Entropy}

The Rényi entropy due to [36], is characterized as

$$
I_{R}(\gamma)=\frac{1}{1-\gamma} \log \left(\int_{0}^{\infty} f^{\gamma}(x) d x\right)
$$

Let us consider

$$
\begin{aligned}
f^{\gamma}(x)= & (\alpha \beta)^{\gamma} k^{\gamma}(x ; \psi) \bar{K}(x ; \psi)^{\gamma(-\alpha-1)} \mathrm{e}^{-\gamma\left[\frac{1-\bar{K}\left(x ; \psi^{\alpha}\right)^{\alpha}}{\bar{K}(x ; \psi)^{\alpha}}\right]} \\
& \times\left\{1-\mathrm{e}^{-\frac{1-\bar{K}(x ; \psi) \alpha}{\bar{K}(x ; \psi)^{\alpha}}}\right\}^{\gamma(\beta-1)} .
\end{aligned}
$$


Expanding Equation (12) as in Section 3.1, the Rényi entropy reduces to

$$
I_{R}(\gamma)=\frac{1}{1-\gamma} \log \left\{\sum_{i=0}^{\infty} \sum_{l=0}^{i} \zeta_{i, l}^{*} \int_{0}^{\infty} k^{\gamma}(x) \bar{K}^{-\{\alpha(\gamma+i-l)-\gamma\}} d x\right\}
$$

where $\zeta_{i, l}^{*}=\frac{(-1)^{i+l}}{i !}(\alpha \beta)^{\gamma}\left(\begin{array}{l}i \\ l\end{array}\right) \sum_{j=0}^{i}(-1)^{j}(j+i)^{i}\left(\begin{array}{c}\gamma(\beta-1) \\ j\end{array}\right)$.

\subsection{Order Statistics}

Assume that $X_{1}, \ldots, X_{n}$ is a random sample (RS) from the OGE2-G . Furthermore, assume that $X_{i: n}$ denote the $i$ th order statistic (OS). Consequently, pdf of $X_{i: n}$ may be interpreted as

$$
\begin{aligned}
f_{i: n}(x) & =\frac{1}{\beta(i, n-i+1)} f(x) F(x)^{i-1}\{1-F(x)\}^{n-i} . \\
& =\frac{1}{\beta(i, n-i+1)} \sum_{j=0}^{n-i}(-1)^{j}\left(\begin{array}{c}
n-i \\
j
\end{array}\right) f(x) F(x)^{j+i-1} .
\end{aligned}
$$

Inserting Equations (1) and (2) in the last equation, and expanding it as in Section 3.1, we get

$$
f_{i: n}(x)=\sum_{j=0}^{n-i} \eta_{j} h_{m}(x)
$$

where

$$
\eta_{j}=\frac{(-1)^{j}}{\beta(i, n-i+1)}\left(\begin{array}{c}
n-i \\
j
\end{array}\right) \sum_{m=0}^{\infty} \xi_{m}^{*}
$$

and

$$
\xi_{m}^{*}=(-1)^{m} \sum_{i, k=0}^{\infty} \sum_{l=0}^{i} \frac{(-1)^{i+k+l}(k+1)^{i} \Gamma\{\beta(i+j)\}}{i ! k ! \Gamma\{\beta(i+j)-k\}}\left(\begin{array}{l}
i \\
l
\end{array}\right)\left(\begin{array}{c}
-\alpha(i+l+1)-1 \\
m
\end{array}\right) .
$$

\subsection{Stress-Strength Reliability}

Supp $X_{1} \sim$ OGE2-G $\left(\alpha, \beta_{1} ; \psi\right)$ and $X_{2} \sim$ OGE2-G $\left(\alpha, \beta_{2} ; \psi\right)$ are two continuous rvs with pdfs $f_{1}(x)$ and $f_{2}(x)$ and cdfs $F_{1}(x)$ and $F_{2}(x)$, therefore the reliability $R$ is supplied via

$$
R=\mathbb{P}\left(X_{1}>X_{2}\right)=\int_{0}^{\infty} f_{1}(x) F_{2}(x) d x .
$$

Theorem 2. Assume that $X_{1}$ and $X_{2}$ are two independent ros established previously with constant parameters $\beta_{1}$ and $\beta_{2}$. Eventually,

$$
R=\beta_{1} \sum_{i=0}^{\infty}\left(\begin{array}{c}
\beta_{1}-1 \\
i
\end{array}\right)(-1)^{i} \Gamma(i+1)-\beta_{1} \sum_{i=0}^{\infty}\left(\begin{array}{c}
\beta_{1}-1 \\
i
\end{array}\right)\left(\begin{array}{c}
\beta_{2} \\
j
\end{array}\right)(-1)^{i+j} \Gamma(i+j+1) .
$$

Proof. Using Equations (1) and (2) in Equation (15), we have

$$
\begin{aligned}
\int_{0}^{\infty} f_{1}(x) F_{2}(x) d x= & \int_{0}^{\infty} \alpha \beta_{1} k(x ; \psi) \bar{K}(x ; \psi)^{-(\alpha+1)} \mathrm{e}^{-\left\{\frac{1-\bar{K}(x ; \psi)^{\alpha}}{\bar{K}(x ; \psi)^{\alpha}}\right\}} \\
& \times \sum_{i=0}^{\infty}\left(\begin{array}{c}
\beta_{1}-1 \\
i
\end{array}\right)(-1)^{i}\left\{\frac{1-\bar{K}(x ; \psi)^{\alpha}}{\bar{K}(x ; \psi)^{\alpha}}\right\}^{i} \\
& \times\left[1-\sum_{j=0}^{\infty}\left(\begin{array}{c}
\beta_{2} \\
j
\end{array}\right)(-1)^{j}\left\{\frac{1-\bar{K}(x ; \psi)^{\alpha}}{\bar{K}(x ; \psi)^{\alpha}}\right\}^{j}\right] d x .
\end{aligned}
$$


Equation (16) follows immediately after solving the integral with any mathematical software.

\subsection{Stochastic Ordering}

Stochastic ordering has indeed been acknowledged as an essential tool for assessing comparative behavior in reliability theory and other disciplines. Assume $X$ and $Y$ be two rvs via cdfs, sfs and pdfs $F_{1}(x)$ and $F_{2}(x), \bar{F}_{1}(x)=1-F_{1}(x)$ and $\bar{F}_{2}(x)=1-F_{2}(x)$, and $f_{1}(x)$ and $f_{2}(x)$, respectively. In the specific planning, the $\mathrm{rv} X_{1}$ is considered to be lower than $X_{2}$ :

1. Stochastic order (symbolized via $X_{1} \leq_{s t} X_{2}$ ) if $\bar{F}_{1}(x) \leq \bar{F}_{2}(x)$ for all $x$;

2. LL ratio order (symbolized via $X_{1} \leq_{l r} X_{1}$ ) if $f_{1}(x) / f_{2}(x)$ is decreasing in $x \geq 0$;

3. Hazard rate order (symbolized via $X_{1} \leq_{h r} X_{2}$ ) if $\bar{F}_{1}(x) / \bar{F}_{2}(x)$ is decreasing in $x \geq 0$;

4. Reversed hazard rate order (symbolized via $X_{1} \leq_{r h r} X_{2}$ ) if $F_{1}(x) / F_{2}(x)$ is decreasing in $x \geq 0$.

All these four stochastic orders studied in (1)-(4) are connected to one another as a result of [37] and the accompanying ramifications apply:

$$
\left(X_{1} \leq_{r h r} X_{2}\right) \Leftarrow\left(X_{1} \leq_{l r} X_{2}\right) \Rightarrow\left(X_{1} \leq_{h r} X_{2}\right) \Rightarrow\left(X_{1} \leq_{s t} X_{2}\right) .
$$

when sufficient conditions are met, the OGE2-G distributions are ordered with regard to the strongest LL ratio ordering, as shown by the next theorem.

Theorem 3. Assume $X_{1} \sim \operatorname{OGE} 2\left(\alpha_{1}, \beta ; \psi\right)$ and $X_{2} \sim \operatorname{OGE} 2\left(\alpha_{2}, \beta ; \psi\right)$. If $\alpha_{1}<\alpha_{2}$, then $X_{1} \leq_{l r} X_{2}$.

Proof. First, we have the ratio

$$
\frac{f_{1}(x)}{f_{2}(x)}=\frac{\alpha_{1} \beta k(x) \bar{K}^{-\alpha_{1}-1} \mathrm{e}^{-\left\{\frac{1-\bar{K}^{\alpha_{1}}}{\bar{K}^{\alpha_{1}}}\right\}}\left[1-\mathrm{e}^{-\left\{\frac{1-\bar{K}^{\alpha_{1}}}{\bar{K}_{1}}\right\}}\right]^{\beta-1}}{\alpha_{2} \beta k(x) \bar{K}^{-\alpha_{2}-1} \mathrm{e}^{-\left\{\frac{1-\bar{K}^{\alpha_{2}}}{\bar{K}^{\alpha_{2}}}\right\}}\left[1-\mathrm{e}^{-\left\{\frac{1-\bar{K}^{\alpha_{2}}}{\bar{K}^{\alpha_{2}}}\right\}}\right]^{\beta-1}} .
$$

After simplification, we obtain

$$
\frac{f_{1}(x)}{f_{2}(x)}=\frac{\alpha_{1} \bar{K}^{\left(\alpha_{2}-\alpha_{1}\right)} \mathrm{e}^{-\left\{\frac{1-\bar{K}^{\alpha_{1}}}{\bar{K}^{\alpha_{1}}}\right\}+\left\{\frac{1-\bar{K}^{\alpha_{2}}}{\bar{K}^{\alpha_{2}}}\right\}}\left[1-\mathrm{e}^{\left.-\left\{\frac{1-\bar{K}^{\alpha_{1}}}{\bar{K}^{\alpha_{1}}}\right\}\right]^{\beta-1}}\right.}{\alpha_{2}\left[1-\mathrm{e}^{-\left\{\frac{1-\bar{K}^{\alpha_{1}}}{\bar{K}^{\alpha} 1}\right\}}\right]^{\beta-1}} .
$$

Next,

$$
\begin{aligned}
\log \left[\frac{f_{1}(x)}{f_{2}(x)}\right]= & -\log \left(\alpha_{2}-\alpha_{1}\right)+\left(\alpha_{2}-\alpha_{1}\right) \log (\bar{K})-\left\{\frac{1-\bar{K}^{\alpha_{1}}}{\bar{K}^{\alpha_{1}}}\right\}+\left\{\frac{1-\bar{K}^{\alpha_{2}}}{\bar{K}^{\alpha_{2}}}\right\} \\
& +(\beta-1) \log \left[1-\mathrm{e}^{\left.-\left\{\frac{1-\bar{K}^{\alpha_{1}}}{\bar{K}^{\alpha_{1}}}\right\}\right]}\right. \\
& -(\beta-1) \log \left[1-\mathrm{e}^{\left.-\left\{\frac{1-\bar{K}^{\alpha_{2}}}{\bar{K}^{\alpha_{2}}}\right\}\right] .}\right.
\end{aligned}
$$

If $a_{1}<a_{2}$, we obtain 


$$
\begin{aligned}
\frac{d}{d x} \log \left[\frac{f_{1}(x)}{f_{2}(x)}\right]= & \left(\alpha_{2}-\alpha_{1}\right) k(x) \bar{K}^{-\left(\alpha_{1}+\alpha_{2}\right)}+(\beta-1) k(x) \bar{K}^{-1} \\
& \times\left[\frac{\alpha_{1} \bar{k}^{-\alpha_{1}}}{\mathrm{e}^{-\left\{-\frac{1-\overline{-}^{\alpha_{1}}}{\bar{K}^{\alpha}}\right\}}-1}-\frac{\alpha_{2} \bar{K}^{-\alpha_{2}}}{\mathrm{e}^{-\left\{-\frac{1-\bar{K}^{\alpha_{2}}}{\bar{K}^{\alpha_{2}}}\right\}}-1}\right]<0 .
\end{aligned}
$$

Thus, $f_{1}(x) / f_{2}(x)$ is decreasing in $x$ and hence $X_{1} \leq_{l r} X_{2}$.

\section{OGE2-Fréchet Distribution}

In this section, we study the first special model defined in Section 2.2, the OGE2Fréchet (OGE2Fr), in view of its practical application.

The OGE2Fr model can be defined from (1) by taking $K(x ; \psi)=\mathrm{e}^{-(a / x)^{b}}$ and $k(x ; \psi)=$ $b a^{b} x^{-(b+1)} \mathrm{e}^{-(a / x)^{b}}$, as cdf and pdf of the baseline Fréchet distribution with $a, b>0$, respectively. The cdf and pdf of OGE2Fr distribution are, respectively, given by

$$
F(x ; \alpha, \beta, a, b)=\left[1-\mathrm{e}^{1-\left\{1-\mathrm{e}^{-\left(\frac{a}{x}\right)^{b}}\right\}^{-\alpha}}\right]^{\beta}, x>0 \quad \alpha, \beta, a, b>0,
$$

and

$$
\begin{aligned}
f(x ; \alpha, \beta, a, b)= & a^{b} \alpha b \beta x^{-b-1} \mathrm{e}^{1-\left(\frac{a}{x}\right)^{b}-\left(1-\mathrm{e}^{-\left(\frac{a}{x}\right)^{b}}\right)^{-\alpha}}\left(1-\mathrm{e}^{-\left(\frac{a}{x}\right)^{b}}\right)^{-\alpha-1} \\
& \times\left[1-\mathrm{e}^{1-\left(1-\mathrm{e}^{-\left(\frac{a}{x}\right)^{b}}\right)^{-\alpha}}\right]^{\beta-1},
\end{aligned}
$$

where $\alpha, \beta$ and $b$ are shape parameters while $a$ is scale parameter.

The hrf and qf of the OGE2Fr distribution are obtained as

$$
\begin{aligned}
& h(x)= a^{b} \alpha b \beta x^{-b-1} \mathrm{e}^{1-\left(\frac{a}{x}\right)^{b}-\left\{1-\mathrm{e}^{-\left(\frac{a}{x}\right)^{b}}\right\}^{-\alpha}}\left[1-\mathrm{e}^{-\left(\frac{a}{x}\right)^{b}}\right]^{-\alpha-1} \\
& \times {\left[1-\mathrm{e}^{1-\left\{1-\mathrm{e}^{-\left(\frac{a}{x}\right)^{b}}\right\}^{-\alpha}}\right]^{1-\beta}\left[1-\left\{1-\mathrm{e}^{1-\left(1-\mathrm{e}^{-\left(\frac{a}{x}\right)^{b}}\right)^{-\alpha}}\right\}\right.} \\
& Q(u)=\left[a\left\{-\log \left(1-\left[1-\log \left(1-u^{1 / \beta}\right)\right]^{-1 / \alpha}\right)\right\}\right]^{-1 / b} .
\end{aligned}
$$

Figure 1 depicts a visualisation of the pdf and hrf functions, exhibiting the range of shapes that all these functions can take at random input parametric values. The OGE2Fr distribution's pdf can be gradually decreasing, unimodal, and right-skewed, with different curves, tail, and asymmetric aspects, as shown in Figure 1. The hrf, on the other hand, offers an extensive range of increasing, decreasing, unimodal, and increasing-decreasingincreasing (IDI) forms. Given a wide variety of hrf shapes being offered, the OGE2Fr distribution can in fact be a useful tool to model unpredictable time-to-event phenomena.

\subsection{Linear Representation and Related Properties}

The cdf of the OGE2Fr distribution is quite straightforward and is achieved by using the result defined in Equation (6) as

$$
F(x)=F(x: \alpha, \beta, a, b)=\sum_{\ell=0}^{\infty} \xi_{\ell}\left[\mathrm{e}^{-(a / x)^{b}}\right]^{\ell} \quad \ell \geq 1,
$$


where $\ell$ is the power parameter and noting that $\sum_{\ell=0}^{\infty} \xi_{\ell}$ is unity.

For simplicity, we can rewrite the above result as

$$
F(x)=F(x: \alpha, \beta, a, b)=\sum_{\ell=0}^{\infty} \xi_{\ell} \mathrm{e}^{-\ell(a / x)^{b}} .
$$

By differentiating the last term, we can express the density of OGE2Fr model as follows

$$
f(x: \alpha, \beta, a, b)=\sum_{i, j=1}^{\infty} \xi_{\ell} \Pi(x ; \ell, a, b),
$$

where $\Pi(x ; \ell, a, b)=\ell b a^{b} x^{-b-1} \mathrm{e}^{-\ell\left(\frac{a}{x}\right)^{b}}$ represents the Fréchet density function with power parameter $\ell$. Equation (22) enforces the fact that OGE2Fr density is a linear combination of Fréchet densities. Thus, we can derive various mathematical properties using Fréchet distribution.

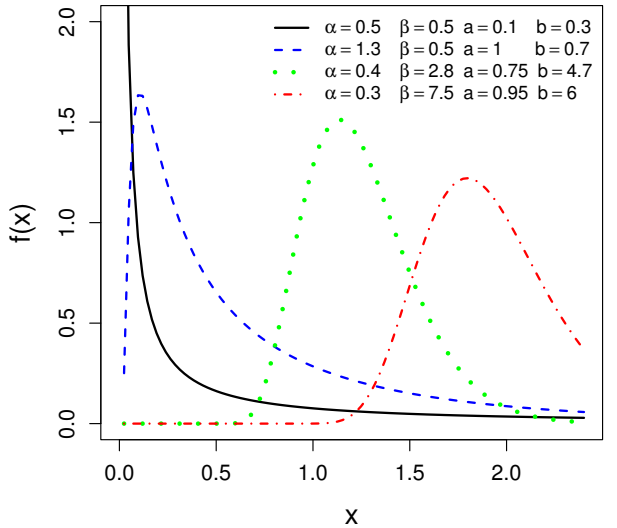

(a)

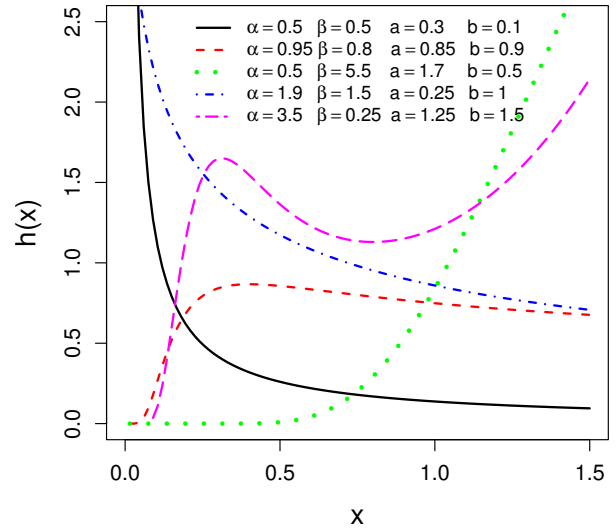

(b)

Figure 1. (a) Plots of density and (b) hazard rate of the OGE2Fr model for some random parameter values.

Moments are the heart and soul of any statistical analysis. Moments can be used to evaluate the most essential characteristics such as mean, variance, skewness, and kurtosis of a distribution. We now directly present the mathematical expressions for the moments of OGE2Fr model as follows. Let $Y_{\ell}$ be a random variable with density $\Pi(x ; \ell, a, b)$. Then, core properties of $X$ can follow from those of $Y_{\ell}$. First, the $p$ th ordinary moment of $X$ can be written as

$$
\mu_{p}^{\prime}=\sum_{i, j=1}^{\infty} \xi_{\ell} \ell a^{p} \frac{\ell^{1-p / b}}{\Gamma(1-p / b)} .
$$

Second, the cumulants $\left(\kappa_{p}\right)$ of $X$ can be determined recursively from (23) as $\kappa_{s}=\mu_{s}^{\prime}-$ $\sum_{k=1}^{s-1}\left(\begin{array}{c}s-1 \\ k-1\end{array}\right) \kappa_{k} \mu_{s-k}^{\prime}$, respectively, where $\kappa_{1}=\mu_{1}^{\prime}$. The skewness $\gamma_{1}=\kappa_{3} / \kappa_{2}^{3 / 2}$ and kurtosis $\gamma_{2}=\kappa_{4} / \kappa_{2}^{2}$ of $X$ can be calculated from the third and fourth standardized cumulants. Plots of mean, variance, skewness and kurtosis of the OGE2Fr distribution are displayed in Figure 2. These plots signifies the significant role of the parameters $\alpha$ and $\beta$ in modeling the behaviors of $X$.

Third, the $p$ th incomplete moment of $X$, denoted by $m_{p}(y)=E\left(X^{p} \mid X \leq y\right)=$ $\int_{0}^{y} x^{p} f_{\mathrm{OGE2Fr}}(x) d x$, is easily found changing variables from the lower incomplete gamma function $\gamma(v, u x)=\int_{0}^{\infty} x^{v-1} \mathrm{e}^{-\mathrm{ux}} \mathrm{dx}$ when calculating the corresponding moment of $Y_{\ell}$. Then, we obtain

$$
m_{p}(y)=\sum_{i, j=1}^{\infty} \xi_{\ell} a^{p} \ell^{1+p / b} \Gamma\left(1-p / b, \ell(a / x)^{b}\right) .
$$


Fourth, the first incomplete moment $m_{1}(z)$ is used to construct the Bonferroni and Lorenz curves as discussed in Section 3.2. Figure 3 provides the income inequality curves (Bonferroni \& Lorenz) of the proposed distribution which can easily be derived from (24), respectively, where $q=Q_{(} \pi$ ) is the qf of $X$ derived from Equation (20).

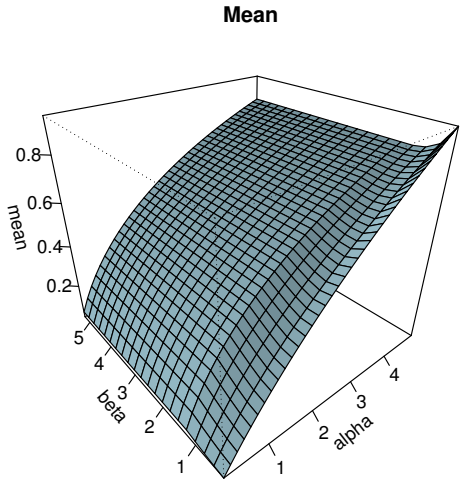

(a)

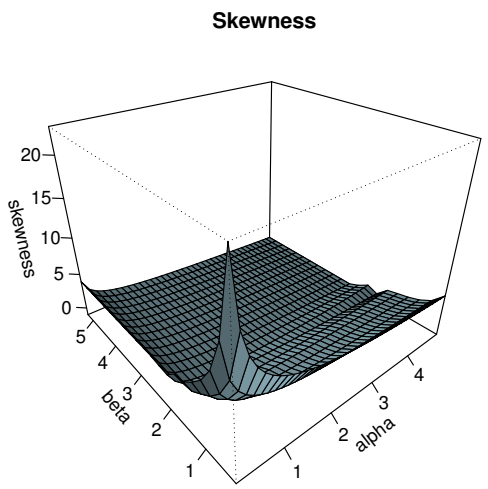

(c)

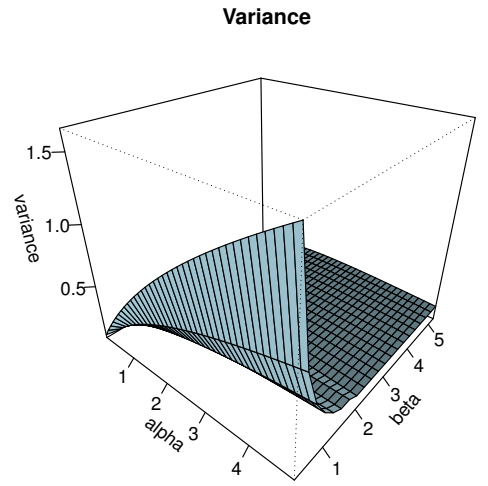

(b)

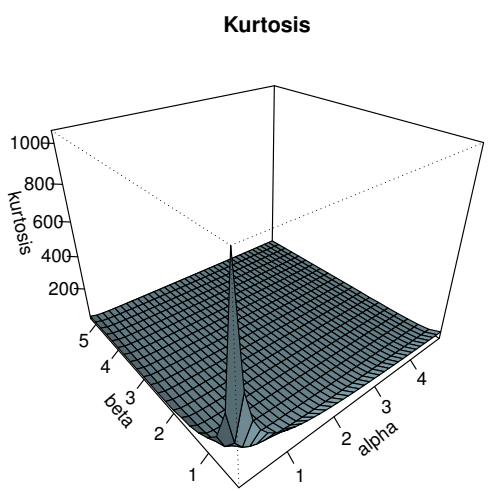

(d)

Figure 2. Plots of (a) mean, (b) variance, (c) skewness and (d) kurtosis of the OGE2Fr distribution.

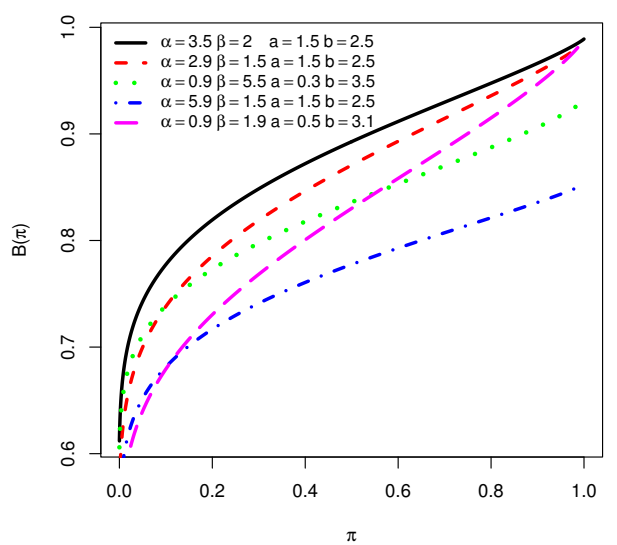

(a)

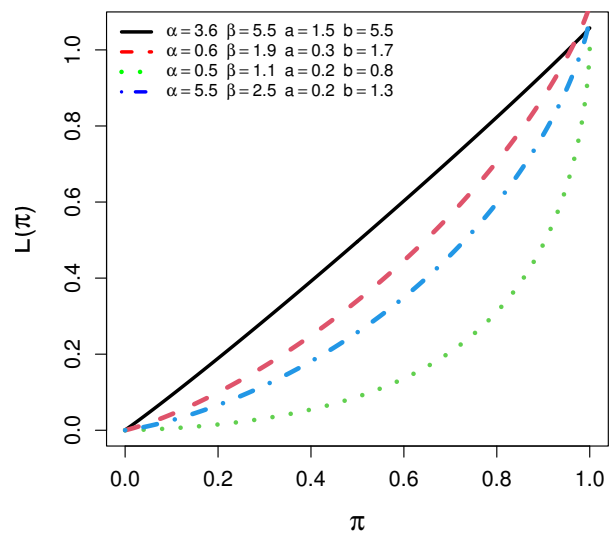

(b)

Figure 3. Plots of (a) Bonferroni curve and (b) Lorenz curve of OGE2Fr model. 


\subsection{Parameter Estimation}

Let $x_{1}, \ldots, x_{n}$ be a sample of size $n$ from the OGE2Fr distribution given in Equation (18). The $\log$-likelihood function $\ell=\ell(\Theta)$ for the vector of parameters $\Theta=(\alpha, \beta, a, b)$ is

$$
\begin{aligned}
\ell= & n b \log (a)+n \log (\alpha \beta b)-(b+1) \sum_{i=1}^{n} \log \left(x_{i}\right)-\sum_{i=1}^{n}\left(\frac{a}{x_{i}}\right)^{b}+\sum_{i=1}^{n}\left[1-\left\{1-\mathrm{e}^{-\left(a / x_{i}\right)^{b}}\right\}^{-\alpha}\right] \\
& -(\alpha+1) \sum_{i=1}^{n} \log \left[1-\mathrm{e}^{-\left(\frac{a}{x_{i}}\right)^{b}}\right]+(\beta-1) \sum_{i=1}^{n} \log \left[1-\mathrm{e}^{1-\left(1-\mathrm{e}^{-\left(\frac{a}{x_{i}}\right)^{b}}\right)^{-\alpha}}\right] .
\end{aligned}
$$

The components of score vectors $\mathrm{U}(\Theta)$ are

$$
\begin{aligned}
& U_{\alpha}=\frac{n}{\alpha}-\sum_{i=1}^{n} \log \left[1-\mathrm{e}^{-\left(\frac{a}{x_{i}}\right)^{b}}\right]+\sum_{i=1}^{n}\left[1-\mathrm{e}^{-\left(\frac{a}{x_{i}}\right)^{b}}\right]^{-\alpha} \log \left[1-\mathrm{e}^{-\left(\frac{a}{x_{i}}\right)^{b}}\right] \\
& +(\beta-1) \sum_{i=1}^{n} \frac{\left\{1-\mathrm{e}^{-\left(\frac{a}{x_{i}}\right)^{b}}\right\}^{-\alpha} \log \left\{1-\mathrm{e}^{-\left(\frac{a}{x_{i}}\right)^{b}}\right\}}{1-\mathrm{e}^{-1+\left\{1-\mathrm{e}^{-\left(\frac{a}{x_{i}}\right)^{b}}\right\}^{-\alpha}},} \\
& U_{\beta}=\frac{n}{\beta}+\sum_{i=1}^{n} \log \left[1-\mathrm{e}^{1-\left\{1-\mathrm{e}^{-\left(\frac{a}{x_{i}}\right)^{b}}\right\}^{-\alpha}}\right] \text {, } \\
& U_{a}=\frac{n b}{a}-\frac{a^{b} b}{a} \sum_{i=1}^{n} x_{i}^{-b}-\frac{a^{b} b(\alpha+1)}{a} \sum_{i=1}^{n} \frac{x_{i}^{-b}}{\mathrm{e}^{\left(\frac{a}{x_{i}}\right)^{b}}-1}-\frac{a^{b} b \alpha}{a} \sum_{i=1}^{n} \frac{x_{i}^{-b}\left\{1-\mathrm{e}^{-\left(\frac{a}{x_{i}}\right)^{b}}\right\}^{-\alpha}}{1-\mathrm{e}^{-\left(\frac{a}{x_{i}}\right)^{b}}} \\
& +\frac{\alpha(\beta-1) a^{b} b}{a} \sum_{i=1}^{n} \frac{x_{i}^{-b}\left\{1-\mathrm{e}^{-\left(\frac{a}{x_{i}}\right)^{b}}\right\}^{-\alpha}}{\left[1-\mathrm{e}^{\left.\left(\frac{a}{x_{i}}\right)^{b}\right]}\right] 1-\mathrm{e}^{\left.-1+\left\{1-\mathrm{e}^{-\left(\frac{a}{x_{i}}\right)^{b}}\right\}^{-\alpha}\right]},}, \\
& U_{b}=\frac{n}{b}+n \log (a)-\sum_{i=1}^{n} \log \left(x_{i}\right)-a^{b} \sum_{i=1}^{n} x_{i}^{-b} \log \left(\frac{a}{x_{i}}\right) \\
& -(\alpha+1) a^{b} \sum_{i=1}^{n} \frac{x_{i}^{-b} \log \left(\frac{a}{x_{i}}\right)}{\mathrm{e}^{\left(\frac{a}{x_{i}}\right)^{b}}-1}+\alpha a^{b} \sum_{i=1}^{n} \frac{x_{i}^{-b} \log \left(\frac{a}{x_{i}}\right)\left\{1-\mathrm{e}^{-\left(\frac{a}{x_{i}}\right)^{b}}\right\}^{-\alpha}}{\mathrm{e}^{\left(\frac{a}{x_{i}}\right)^{b}}-1} \\
& +\alpha a^{b}(\beta-1) \sum_{i=1}^{n} \frac{x_{i}^{-b} \log \left(\frac{a}{x_{i}}\right)\left\{1-\mathrm{e}^{\left.-\left(\frac{a}{x_{i}}\right)^{b}\right\}^{-\alpha}}\right.}{\left[\mathrm{e}^{\left(\frac{a}{x_{i}}\right)^{b}}-1\right]\left[1-\mathrm{e}^{\left.-1+\left\{1-\mathrm{e}^{-\left(\frac{a}{x_{i}}\right)^{b}}\right\}^{-\alpha}\right]} .\right.}
\end{aligned}
$$

\subsection{Order Statistics}

For a random sample of $X_{1}, \ldots, X_{n}$ taken from the OGE2Fr distribution with $X_{i: n}$ as the $i$ th order statistic. For $i=1,2,3, \ldots, n$, the pdf corresponding to $X_{i: n}$ can be expressed as 


$$
\begin{aligned}
f_{i: n}(x) & =\frac{1}{\beta(i, n-i+1)} f(x) F(x)^{i-1}\{1-F(x)\}^{n-i} . \\
& =\frac{1}{\beta(i, n-i+1)} \sum_{j=0}^{n-i}(-1)^{j}\left(\begin{array}{c}
n-i \\
j
\end{array}\right) f(x) F(x)^{j+i-1},
\end{aligned}
$$

where $f(x)$ and $F(x)$ are the pdf and cdf of OGE2Fr distribution, respectively. Inserting Equations (17) and (18), and using the result defined in Section 3, we have

$$
f_{i: n}(x ; m a, b)=\sum_{j=0}^{n-i} \eta_{j} m b a^{b} x^{-b-1} \mathrm{e}^{-m(a / x)^{b}},
$$

where

$$
\eta_{j}=\frac{(-1)^{j}}{\beta(i, n-i+1)}\left(\begin{array}{c}
n-i \\
j
\end{array}\right) \sum_{m=0}^{\infty} \xi_{m}^{*}
$$

and

$$
\xi_{m}^{*}=(-1)^{m} \sum_{i, k=0}^{\infty} \sum_{l=0}^{i} \frac{(-1)^{i+k+l}(k+1)^{i} \Gamma\{\beta(i+j)\}}{i ! k ! \Gamma\{\beta(i+j)-k\}}\left(\begin{array}{l}
i \\
l
\end{array}\right)\left(\begin{array}{c}
-\alpha(i+l+1)-1 \\
m
\end{array}\right) .
$$

$f_{i: n}(x ; m a, b)$ is the probability density function of the OGE2Fr distribution with parameters $m a$ and $b$.

\subsection{Stochastic Ordering}

In several areas of probability and statistics, stochastic ordering and disparities are being adhered to at an accelerating rate. For example, in analyzing the contrast of investment returns to random cash flows; two manufacturers may use distinct technologies to make gadgets with the same function, resulting in non-identical life distributions or comparing the strength of dependent structures. Here, we use the term stochastic ordering to refer to any ordering relation on a space of probability measures in a wide sense. Let $X$ and $Y$ be two rvs from OGE2Fr distributions, with assumptions previously mentioned in Section 3. Given that $a_{1}<a_{2}$, and for $X_{1} \leq_{l r} X_{2}, f_{1}(x) / f_{2}(x)$ shall be decreasing in $x$ if and only if the following result holds Let $X$ and $Y$ be two rvs from OGE2Fr distributions, with assumptions previously mentioned in Section 3. Given that $a_{1}<a_{2}$, and for $X_{1} \leq_{l r} X_{2}$, $f_{1}(x) / f_{2}(x)$ shall be decreasing in $x$ if the following result holds

$$
\begin{aligned}
\frac{d}{d x} \log \left[\frac{f_{1}(x)}{f_{2}(x)}\right]= & \left(\alpha_{2}-\alpha_{1}\right) b a^{b} x^{-(b+1)} \mathrm{e}^{-(a / x)^{b}} 1-\mathrm{e}^{-(a / x)^{b}-\left(\alpha_{1}+\alpha_{2}\right)} \\
& +(\beta-1)\left[b a^{b} x^{-(b+1)} \mathrm{e}^{-(a / x)^{b}}\right]\left(1-\mathrm{e}^{-(a / x)^{b}}\right)^{-1} \\
& \times\left[\frac{\alpha_{1}\left(1-\mathrm{e}^{-(a / x)^{b}}\right)^{-\alpha_{1}}}{\mathrm{e}^{-\left(1-\mathrm{e}^{-(a / x)^{b}}\right)^{-a_{1}}-1}-1}\right] \\
& +(1-\beta)\left[b a^{b} x^{-(b+1)} \mathrm{e}^{-(a / x)^{b}}\right]\left\{1-\mathrm{e}^{-(a / x)^{b}}\right\}^{-1} \\
& \times\left[\frac{\alpha_{1}\left(1-\mathrm{e}^{-(a / x)^{b}}\right)^{-\alpha_{2}}}{\mathrm{e}^{-\left(1-\mathrm{e}^{-(a / x)^{b}}\right)^{-a_{2}}-1}-1}\right]<0
\end{aligned}
$$

\subsection{Simulation Study}

By using the result defined in Equation (25), we evaluate the sensitivity of the method of estimations using the MLEs of OGE2Fr distribution parameters by Monte Carlo simulation technique. The simulation study is conducted for sample sizes $n=$ $50,100,200,300,500,600$ and parameter combinations, denoted by $\omega_{(.)}$, are: 
- $\omega_{1}: \alpha=0.3, \beta=1.7, a=0.8$ and $b=0.5$;

- $\quad \omega_{2}: \alpha=0.5, \beta=1.5, a=0.8$ and $b=0.7$;

- $\quad \omega_{3}: \alpha=1.5, \beta=2.4, a=1.5$ and $b=0.5$.

We use Equation (20) to generate the random observations. For each $\omega_{(.)}$, the empirical bias and MSE values are the average of the values from $N=1000$ simulated samples for given sample size $n$. The formula to evaluate the mean squared error (MSEs) and the average bias (Bias) of each parameter, is given below

$$
\operatorname{MSE}(\hat{\Theta})=\sum_{i=1}^{N} \frac{\left(\hat{\Theta}_{i}-\Theta\right)^{2}}{N} \quad \text { and } \quad \operatorname{Bias}(\hat{\Theta})=\sum_{i=1}^{N} \frac{\hat{\Theta}_{i}}{N}-\Theta .
$$

We report the results of the AE, Bias and MSE for the parameters $\alpha, \beta, a$ and $b$ in Table 2. The MSE of the estimators increases when the assumed model deviates from the genuine model, as anticipated. When the sample size grows larger and the symmetry degrades, the MSE shrinks. Generally speaking, the MSE decreases when the kurtosis grows. Similarly, when the asymmetry rises, the bias grows, and vice versa. As the kurtosis grows, the bias becomes smaller. In conclusion, it is apparent that the MSEs and Biases decrease when the sample size $n$ increases. Thus, we can say that the MLEs perform satisfactorily well in estimating the parameters of the OGE2Fr distribution.

Table 2. AEs, MSEs and Biases for $\omega_{1}, \omega_{2} \& \omega_{3}$.

\begin{tabular}{|c|c|c|c|c|c|c|c|c|c|c|}
\hline & \multicolumn{4}{|c|}{$\omega_{1}$} & \multicolumn{3}{|c|}{$\omega_{2}$} & \multicolumn{3}{|c|}{$\omega_{3}$} \\
\hline & $\theta$ & $\mathrm{AE}$ & MSE & Bias & $\mathrm{AE}$ & MSE & Bias & $\mathrm{AE}$ & MSE & Bias \\
\hline \multirow[t]{4}{*}{$n=50$} & $\alpha$ & 1.14 & 2.267 & 0.982 & 1.97 & 0.997 & 1.787 & 2.14 & 2.892 & 0.447 \\
\hline & $\beta$ & 2.77 & 1.367 & 0.531 & 2.14 & 1.459 & 0.508 & 3.04 & 1.667 & 0.771 \\
\hline & $a$ & 1.70 & 1.119 & 0.539 & 1.45 & 1.517 & 0.621 & 2.12 & 1.486 & 0.541 \\
\hline & $b$ & 1.23 & 0.997 & 0.408 & 1.67 & 0.899 & 0.447 & 1.19 & 1.035 & 0.546 \\
\hline \multirow[t]{4}{*}{$n=100$} & $\alpha$ & 0.65 & 1.892 & 0.877 & 1.88 & 0.901 & 1.205 & 2.02 & 2.793 & 0.420 \\
\hline & $\beta$ & 2.61 & 1.213 & 0.509 & 1.99 & 1.312 & 0.472 & 2.91 & 1.522 & 0.656 \\
\hline & $a$ & 1.31 & 1.092 & 0.511 & 1.11 & 1.349 & 0.555 & 1.93 & 1.397 & 0.502 \\
\hline & $b$ & 0.82 & 0.901 & 0.388 & 1.40 & 0.835 & 0.420 & 1.08 & 0.923 & 0.522 \\
\hline \multirow[t]{4}{*}{$n=300$} & $\alpha$ & 0.52 & 1.407 & 0.853 & 1.56 & 0.866 & 0.773 & 1.72 & 2.771 & 0.407 \\
\hline & $\beta$ & 2.33 & 1.809 & 0.477 & 1.83 & 1.292 & 0.443 & 2.67 & 1.487 & 0.629 \\
\hline & $a$ & 1.19 & 0.934 & 0.483 & 0.92 & 1.293 & 0.507 & 1.86 & 1.203 & 0.488 \\
\hline & $b$ & 0.78 & 0.866 & 0.363 & 1.32 & 0.801 & 0.417 & 0.87 & 0.847 & 0.497 \\
\hline \multirow[t]{4}{*}{$n=400$} & $\alpha$ & 0.39 & 0.775 & 0.639 & 0.89 & 0.519 & 0.651 & 1.55 & 1.636 & 0.374 \\
\hline & $\beta$ & 1.89 & 0.686 & 0.393 & 1.59 & 0.897 & 0.370 & 2.53 & 0.883 & 0.409 \\
\hline & $a$ & 0.93 & 0.473 & 0.411 & 0.85 & 0.945 & 0.477 & 1.59 & 0.860 & 0.359 \\
\hline & $b$ & 0.55 & 0.519 & 0.287 & 0.81 & 0.569 & 0.374 & 0.57 & 0.580 & 0.338 \\
\hline \multirow[t]{4}{*}{$n=500$} & $\alpha$ & 0.32 & 0.701 & 0.497 & 0.54 & 0.298 & 0.455 & 1.48 & 1.475 & 0.325 \\
\hline & $\beta$ & 1.67 & 0.529 & 0.376 & 0.51 & 0.774 & 0.323 & 2.46 & 0.661 & 0.373 \\
\hline & $a$ & 0.81 & 0.355 & 0.337 & 0.83 & 0.670 & 0.435 & 1.52 & 0.663 & 0.323 \\
\hline & $b$ & 0.49 & 0.393 & 0.264 & 0.75 & 0.411 & 0.325 & 0.49 & 0.444 & 0.317 \\
\hline \multirow[t]{4}{*}{$n=600$} & $\alpha$ & 0.31 & 0.472 & 0.316 & 0.51 & 0.252 & 0.424 & 1.45 & 1.437 & 0.311 \\
\hline & $\beta$ & 1.65 & 0.333 & 0.288 & 0.49 & 0.554 & 0.311 & 2.45 & 0.516 & 0.361 \\
\hline & $a$ & 0.80 & 0.271 & 0.298 & 0.80 & 0.655 & 0.409 & 1.50 & 0.577 & 0.305 \\
\hline & $b$ & 0.50 & 0.313 & 0.224 & 0.71 & 0.405 & 0.317 & 0.50 & 0.440 & 0.313 \\
\hline
\end{tabular}

\section{Application of OGE2Fr to Premium Data}

Most skewed distributions are suitable to measure risk measures associated with actuarial data. The risks involve credit, portfolio, capital, premiums losses, and stocks prices among others. We focus our attention on the stakes based on premiums. Premiums are the payments for insurance that the customer pay to the company to which they are insured. In this section, we apply the OGE2Fr lifetime model for the statistical analysis of 
two real life data sets both of which include premium losses. Our aim is to compare the fits of the OGE2Fr model with other well-known generalizations of the Fréchet (Fr) models given in Table 3.

The first premium data set, designated as PD1, is derived from complaints upheld against vehicle insurance firms as a proportion of their overall business over a two-year period. The study was conducted by DFR (Darla Fry Ross) insurance and investment company (2009-2016), registered in New York state. The most common complaints are over delays in the settlement of no-fault claims and non-renewal of insurance. Top of the list are insurers with the fewest upheld complaints per million USD of premiums. The companies with the greatest complaint ratios are at the bottom of the list. The data understudy is from the year 2016. The second premium data, denoted by PD2, signifies the net premiums written (in billions of USD) to insurers which, under Article 41 of the New York Insurance Law, are required to meet minimum financial security requirements. Table 4: Descriptives statistics of PD1 and PD2.

Table 3. The comparative fitted models.

\begin{tabular}{ccc}
\hline Distribution & Author(s) & $\boldsymbol{\Theta}$ \\
\hline BFr & Nadarajah and Gupta [38]; Barreto and Souza [39] & $(\alpha, \beta, \mathrm{a}, \mathrm{b})$ \\
KwFr & Cordiero et al. [40] & $(\alpha, \beta, \mathrm{a}, \mathrm{b})$ \\
EGFr & Cordiero et al. [41] & $(\alpha, \beta, \mathrm{a}, \mathrm{b})$ \\
MOFr & Krishna et al. [42] & $(\theta, \mathrm{a}, \mathrm{b})$ \\
EFr & Nadarajah and Kotz [43] & $(\theta, \mathrm{a}, \mathrm{b})$ \\
GaFr & Da Silva et al. [44] & $(\gamma, \mathrm{a}, \mathrm{b})$ \\
TLFr & Abbas et al. [45] & $(\theta, \mathrm{a}, \mathrm{b})$ \\
OLiFr & Silva et al. [29] & $(\lambda, \mathrm{a}, \mathrm{b})$ \\
Fr & Fréchet [46] & $(\mathrm{a}, \mathrm{b})$ \\
\hline
\end{tabular}

Table 4. The descriptive statistics related to PD1 \& PD2.

\begin{tabular}{cccccccc}
\hline Data & Sample Size & $\mu$ & $\sigma$ & Lowest & Highest & Skewness & Kurtosis \\
\hline PD1 & 89 & 14.08 & 638.38 & 1.048 & 204.17 & 5.31 & 34.97 \\
PD2 & 34 & 3629.40 & $59,372,161$ & 7.567 & $36,502.53$ & 3.06 & 9.18 \\
\hline
\end{tabular}

The OGE2Fr model is validated through the discriminatory criterions (DCs) we considered for each data set. It includes the negative log-likelihood $(\hat{-\ell})$ of the model taken at the corresponding MLEs, the Akaike Information Criterion (AICs), Bayesian Information Criterion (BICs), Anderson-Darling (AD), Cramér-von Mises (CvM), and Kolmogrov-Smirnov (KS) as well as the $p$-value (P-KS) of the related KS test. We use the method of maximum likelihood estimation to estimate the unknown parameters as presented in Section 4.2. For each criterion (except $p$-value (KS)) with highest value), the smallest values is gained by the OGE2Fr model, indicating the best fit among its competitive models.

Some descriptive statistics related to these data are given in Table 4 . The skewness and kurtosis are indicative of exponentially tailed data (reversed-J shape). The TTT plots for the both data sets are given in Figure 4. In particular, the TTT plots show largely decreasing hrf, permitting to fit OGE2Fr model on these data sets. The estimated hrf in Figure 5 matches Figure 4. In Table 5, we present the estimates (MLEs) along with their respective standard errors(SEs) while the DCs are listed in Table 6 for PD1 \& PD2, respectively. For a more visual view, the estimated pdf, cdf, sf and Q-Q plots of the OGE2Fr model for two data sets are displayed in Figures 6 and 7. Furthermore, the PP-plots of OGE2Fr and its three other competitive 4-parameter models for PD1 and PD2 are displayed in Figures 8 and 9. The log-likelihood function profiles for PD1 and PD2, respectively, are provided in Figures 10 and 11 to highlight the universality of the MLEs of $\Theta$ vector. The graphical visualizations are indicative of nice fits for the OGE2Fr model. 


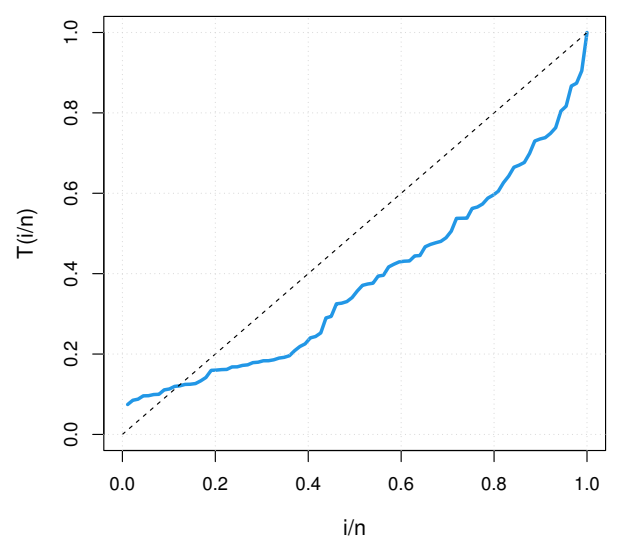

(a)

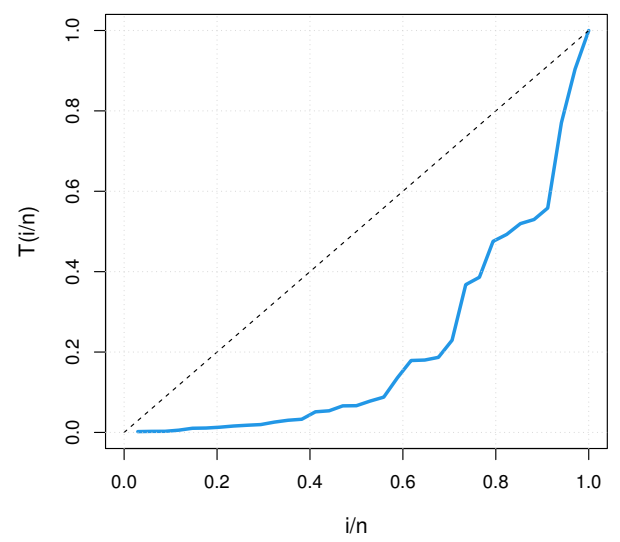

(b)

Figure 4. TTT plots of (a) PD1 and (b) PD2.

Table 5. Estimates and standard errors for PD1 \& PD2.

\begin{tabular}{|c|c|c|c|c|c|}
\hline Distribution & $\Theta$ & MLE(PD1) & SE(PD1) & MLE(PD2) & SE(PD2) \\
\hline \multirow[t]{4}{*}{ OGE2Fr } & $\alpha$ & 0.065 & 0.004 & 2.202 & 0.649 \\
\hline & $\beta$ & 1.177 & 0.145 & 0.298 & 0.143 \\
\hline & $a$ & 2.336 & 0.099 & 24.064 & 19.485 \\
\hline & $b$ & 5.685 & 0.109 & 0.327 & 0.124 \\
\hline \multirow{4}{*}{$\mathrm{BFr}$} & $\alpha$ & 5.623 & 4.692 & 30.671 & 13.743 \\
\hline & $\beta$ & 1.699 & 1.324 & 34.622 & 16.797 \\
\hline & $a$ & 0.855 & 0.646 & 11.391 & 9.267 \\
\hline & $b$ & 0.759 & 0.453 & 0.175 & 0.126 \\
\hline \multirow[t]{4}{*}{$\mathrm{KwFr}$} & $\alpha$ & 6.581 & 4.779 & 6.282 & 3.189 \\
\hline & $\beta$ & 1.409 & 1.161 & 15.235 & 4.679 \\
\hline & $a$ & 0.606 & 0.496 & 5.735 & 3.726 \\
\hline & $b$ & 0.858 & 0.390 & 0.157 & 0.124 \\
\hline \multirow[t]{4}{*}{ EGFr } & $\alpha$ & 12.927 & 9.199 & 6.758 & 5.059 \\
\hline & $\beta$ & 62.076 & 36.099 & 53.635 & 39.338 \\
\hline & $a$ & 21.399 & 17.982 & 22.629 & 16.084 \\
\hline & $b$ & 0.158 & 0.119 & 0.106 & 0.091 \\
\hline \multirow[t]{3}{*}{ MOFr } & $\theta$ & 105.267 & 67.873 & 105.566 & 88.076 \\
\hline & $a$ & 0.060 & 0.028 & 0.302 & 0.224 \\
\hline & $b$ & 14.866 & 0.177 & 0.723 & 0.107 \\
\hline \multirow[t]{3}{*}{ EFr } & $\theta$ & 1.409 & 1.161 & 1.097 & 0.554 \\
\hline & $a$ & 5.442 & 4.611 & 167.306 & 163.093 \\
\hline & $b$ & 0.858 & 0.391 & 0.452 & 0.128 \\
\hline \multirow[t]{3}{*}{$\mathrm{GaFr}$} & $\gamma$ & 5.642 & 3.889 & 14.715 & 5.016 \\
\hline & $a$ & 0.186 & 0.460 & 0.008 & 0.002 \\
\hline & $b$ & 2.060 & 0.769 & 1.603 & 0.395 \\
\hline \multirow[t]{3}{*}{ TLFr } & $\theta$ & 0.075 & 0.009 & 0.122 & 0.098 \\
\hline & $a$ & 53.724 & 46.329 & 78.317 & 54.389 \\
\hline & $b$ & 0.953 & 0.114 & 0.425 & 0.089 \\
\hline \multirow[t]{3}{*}{ OLiFr } & $\lambda$ & 23.260 & 14.666 & 35.788 & 8.969 \\
\hline & $a$ & 6.036 & 0.653 & 8.583 & 1.458 \\
\hline & $b$ & 0.274 & 0.049 & 0.124 & 0.050 \\
\hline \multirow[t]{2}{*}{$\mathrm{Fr}$} & $a$ & 4.178 & 0.564 & 10.630 & 3.207 \\
\hline & $b$ & 1.042 & 0.087 & 0.469 & 0.061 \\
\hline
\end{tabular}




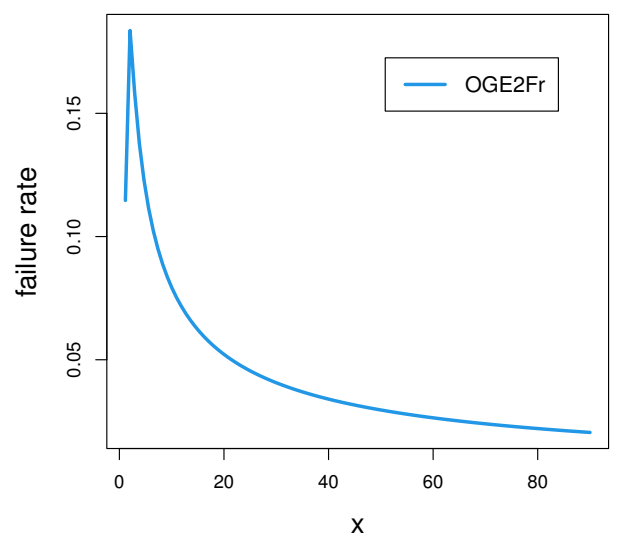

(a)

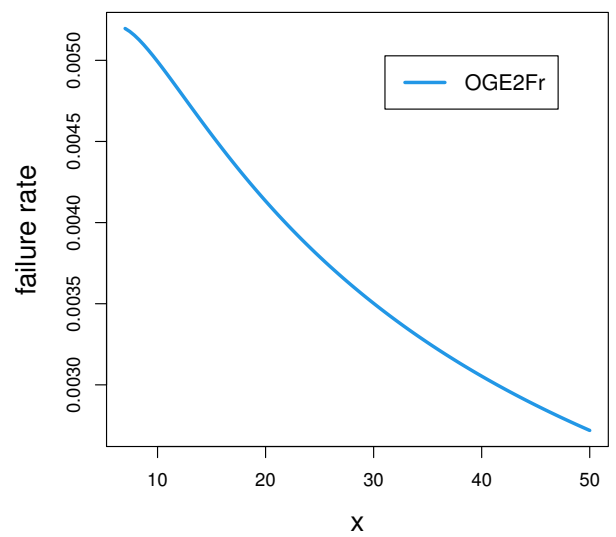

(b)

Figure 5. Estimated hazard rate plots of (a) PD1 and (b) PD2 of OGE2Fr.

Table 6. The statistics $\hat{\ell}$, AIC, CAIC, BIC, HQIC, AD, CvM, KS and $p$-value (KS) for the PD1 \& PD2.

\begin{tabular}{cccccccccc}
\hline Distribution & $\hat{\ell}$ & AIC & CAIC & BIC & HQIC & AD & CvM & KS & $p$-Value (KS) \\
\hline Premium data 1 & & & & & & & & & \\
OGE2Fr & 302.22 & 612.44 & 612.92 & 622.39 & 616.45 & 0.38 & 0.077 & 0.074 & 0.69 \\
BFr & 306.63 & 621.25 & 621.72 & 631.23 & 625.27 & 0.86 & 0.158 & 0.093 & 0.39 \\
KwFr & 306.70 & 621.40 & 621.85 & 631.35 & 625.42 & 0.88 & 0.164 & 0.092 & 0.41 \\
EGFr & 306.76 & 621.51 & 621.99 & 631.47 & 625.52 & 0.84 & 0.152 & 0.098 & 0.34 \\
MOFr & 311.09 & 628.17 & 628.46 & 635.64 & 631.18 & 1.12 & 0.178 & 0.110 & 0.22 \\
EFr & 306.70 & 619.40 & 619.69 & 626.86 & 622.41 & 0.88 & 0.164 & 0.092 & 0.41 \\
GaFr & 305.72 & 617.44 & 617.72 & 624.90 & 620.44 & 0.77 & 0.146 & 0.090 & 0.45 \\
TLFr & 305.65 & 617.29 & 617.58 & 624.76 & 620.30 & 0.71 & 0.133 & 0.090 & 0.43 \\
OLiFr & 308.403 & 622.81 & 623.09 & 630.24 & 625.80 & 0.981 & 0.140 & 0.116 & 0.17 \\
Fr & 306.79 & 617.58 & 617.72 & 622.56 & 619.59 & 0.95 & 0.180 & 0.094 & 0.39 \\
Premium data 2 & & & & & & & & & \\
OGE2Fr & 286.09 & 580.18 & 581.56 & 586.29 & 582.26 & 0.18 & 0.027 & 0.083 & 0.96 \\
BFr & 287.43 & 582.87 & 584.25 & 588.97 & 584.95 & 0.29 & 0.042 & 0.101 & 0.84 \\
KwFr & 287.45 & 582.89 & 584.27 & 588.99 & 584.97 & 0.30 & 0.044 & 0.100 & 0.86 \\
EGFr & 288.21 & 584.41 & 585.79 & 590.52 & 586.50 & 0.39 & 0.053 & 0.102 & 0.84 \\
MOFr & 288.66 & 583.32 & 584.11 & 587.89 & 584.86 & 0.40 & 0.060 & 0.103 & 0.83 \\
EFr & 288.70 & 583.40 & 584.20 & 587.98 & 584.96 & 0.47 & 0.063 & 0.119 & 0.68 \\
GaFr & 287.62 & 581.24 & 582.04 & 585.82 & 582.81 & 0.33 & 0.045 & 0.101 & 0.85 \\
TLFr & 287.70 & 581.39 & 582.19 & 585.97 & 582.96 & 0.35 & 0.047 & 0.102 & 0.84 \\
OLiFr & 287.52 & 581.54 & 581.84 & 586.62 & 582.60 & 0.310 & 0.077 & 0.094 & 0.894 \\
Fr & 288.79 & 581.58 & 581.97 & 584.63 & 582.62 & 0.49 & 0.065 & 0.104 & 0.82 \\
\hline
\end{tabular}

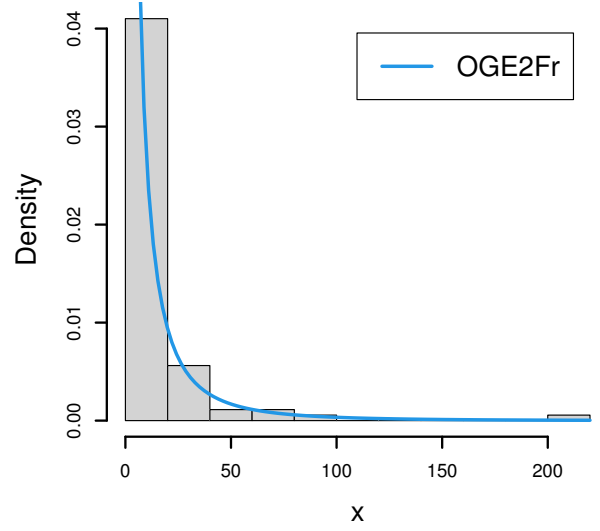

(a)

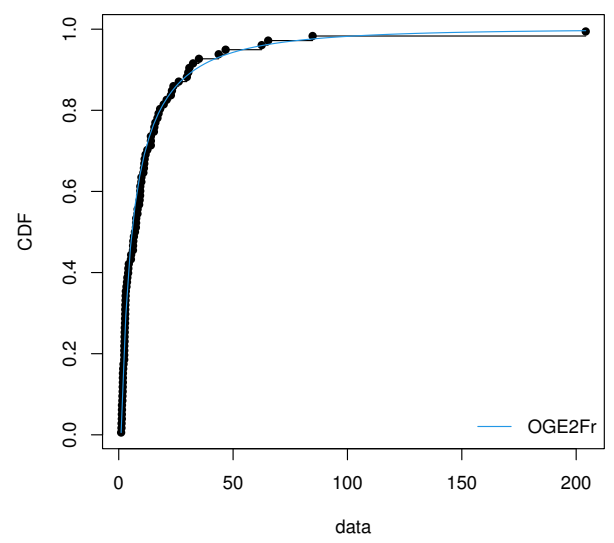

(b)

Figure 6. Cont. 


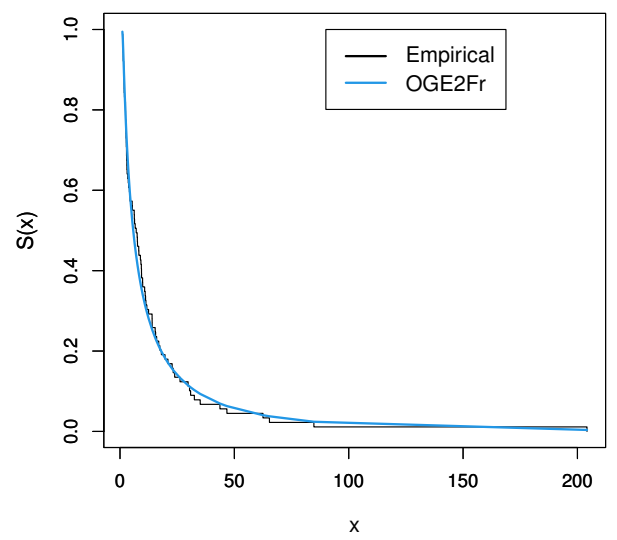

(c)

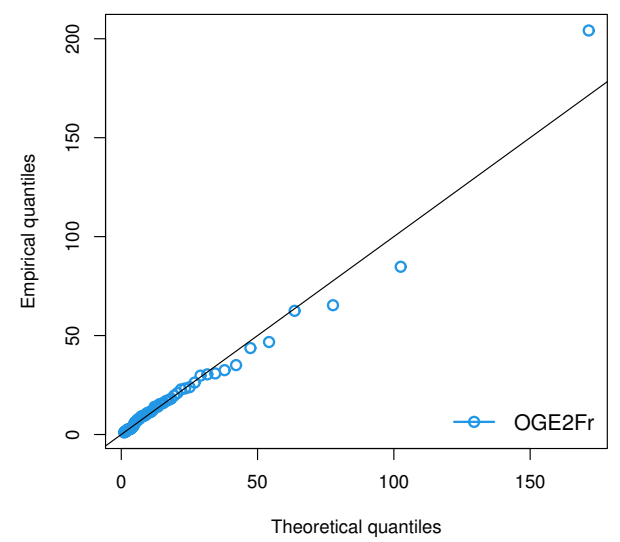

(d)

Figure 6. Estimated (a) density, (b) cdf, (c) sf, and (d) QQ-plot for PD1.

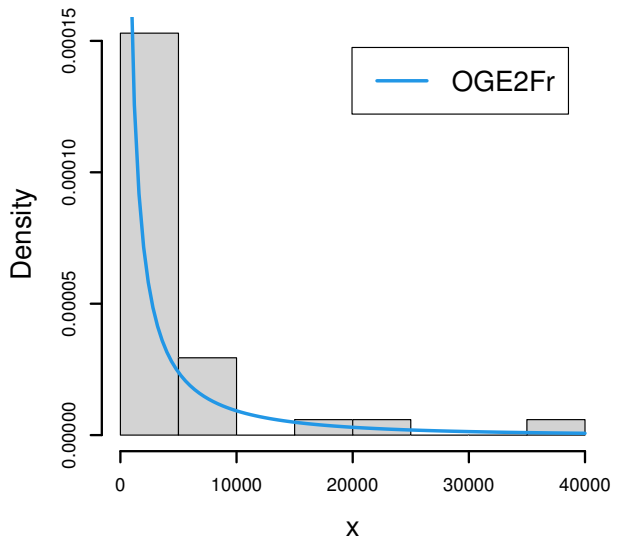

(a)

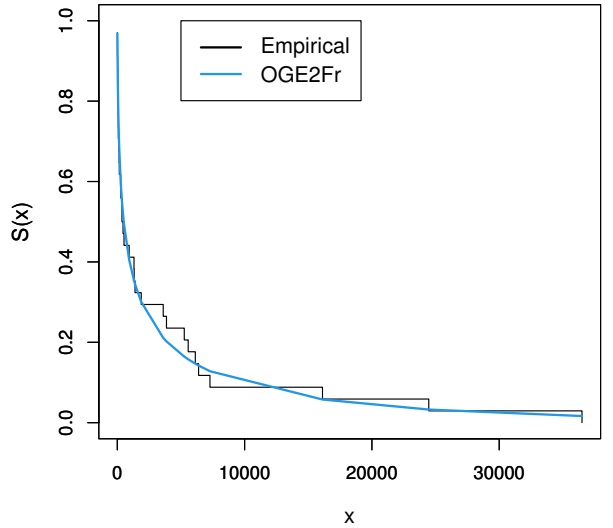

(c)

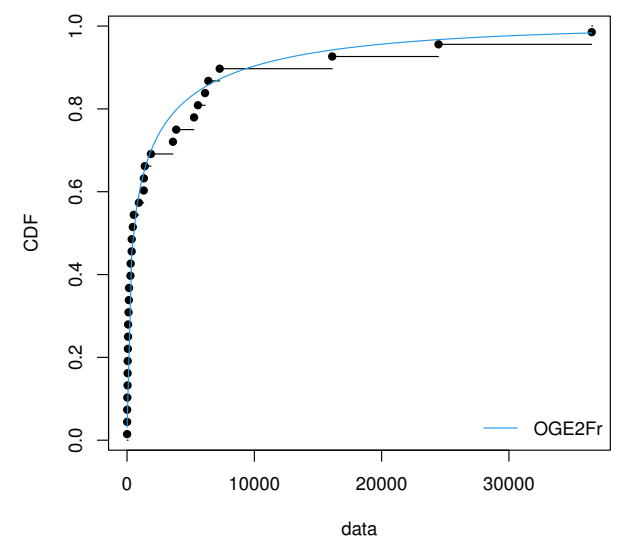

(b)

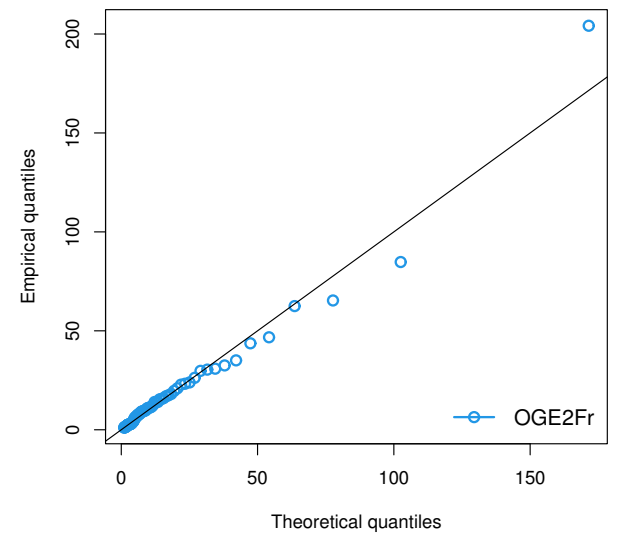

(d)

Figure 7. Estimated (a) density (b) cdf (c) sf, and (d) QQ-plot for PD2. 


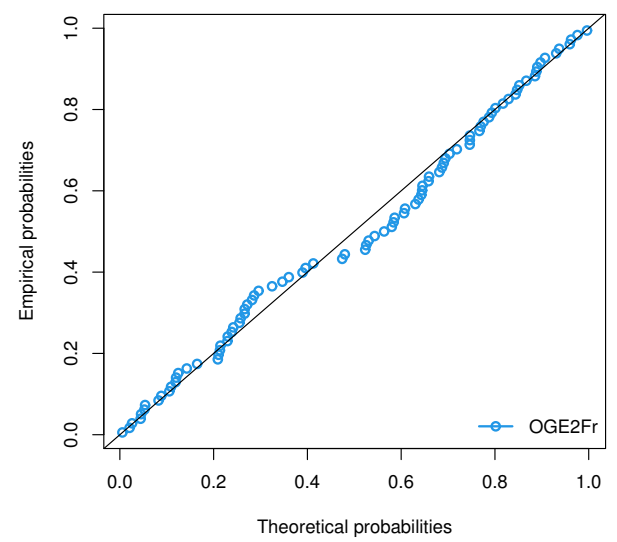

(a)

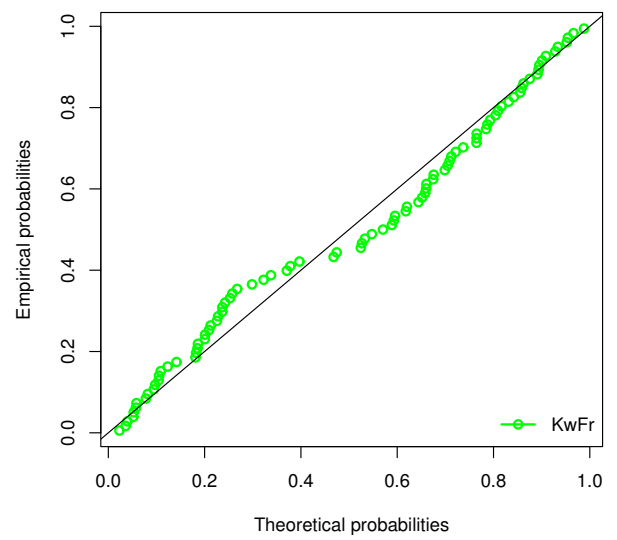

(c)

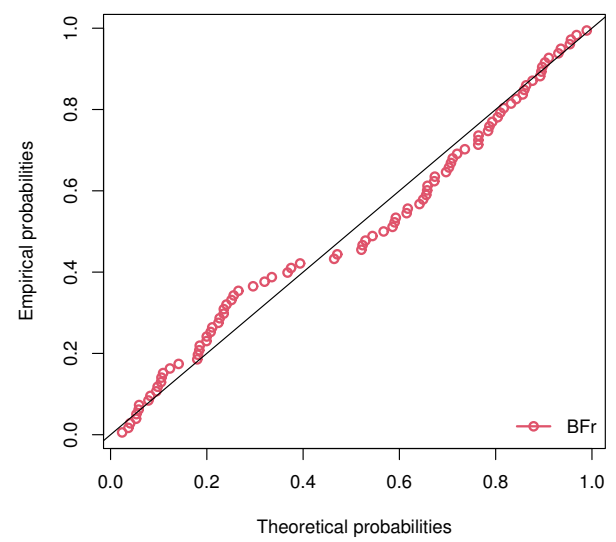

(b)

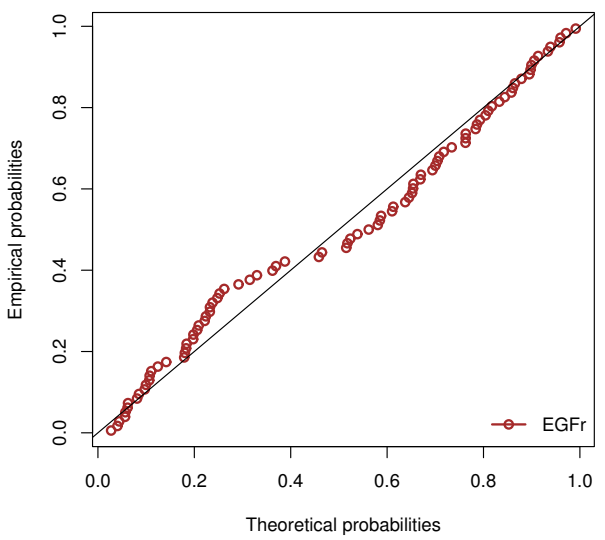

(d)

Figure 8. PP-plots of (a) OGE2Fr alongside competitive (b) BFr, (c) KwFr and (d) EGFr (4-parameter models) for PD1.

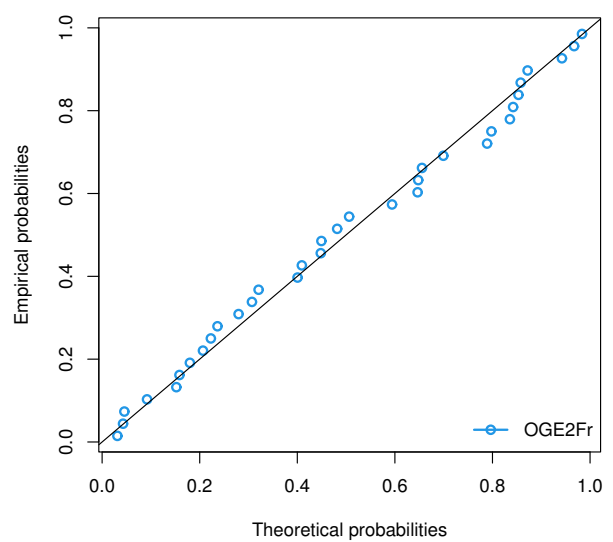

(a)

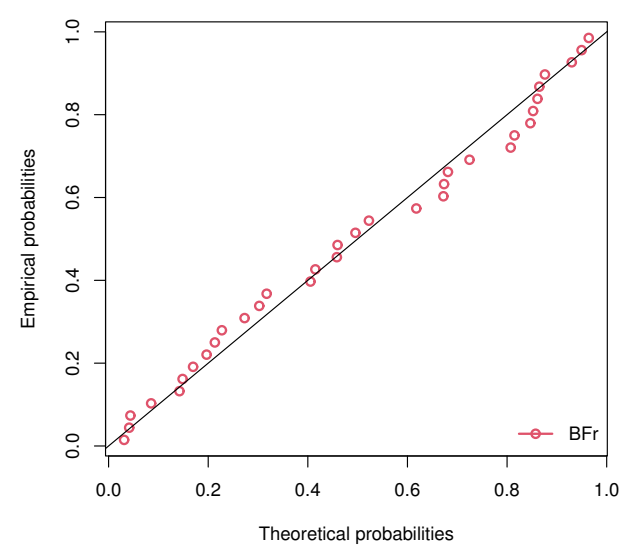

(b)

Figure 9. Cont. 


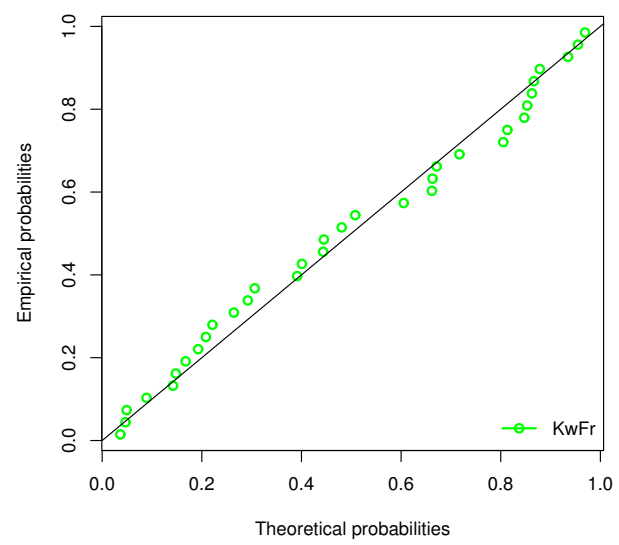

(c)

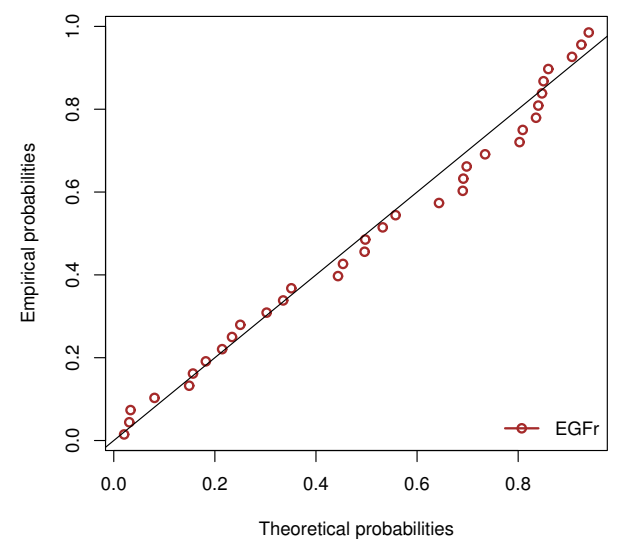

(d)

Figure 9. PP-plots of (a) OGE2Fr alongside competitive (b) BFr, (c) KwFr and (d) EGFr (4-parameter models) for PD2.

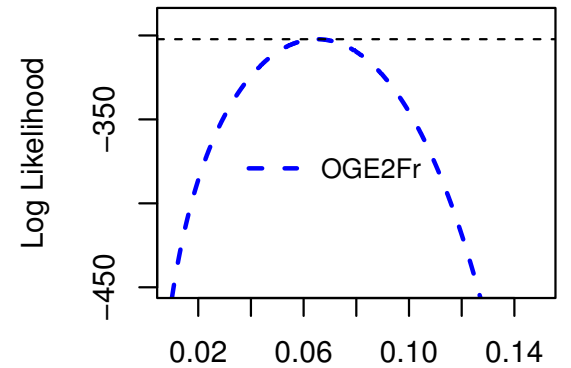

$\alpha$

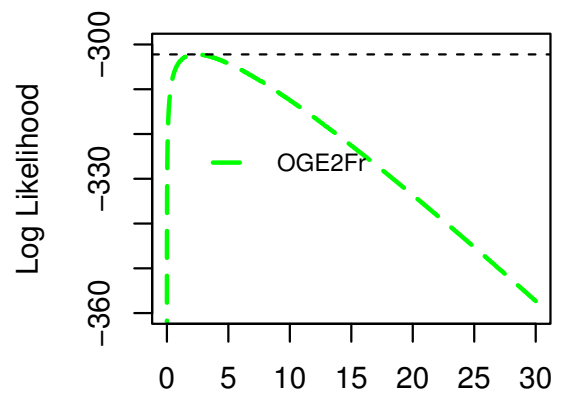

a
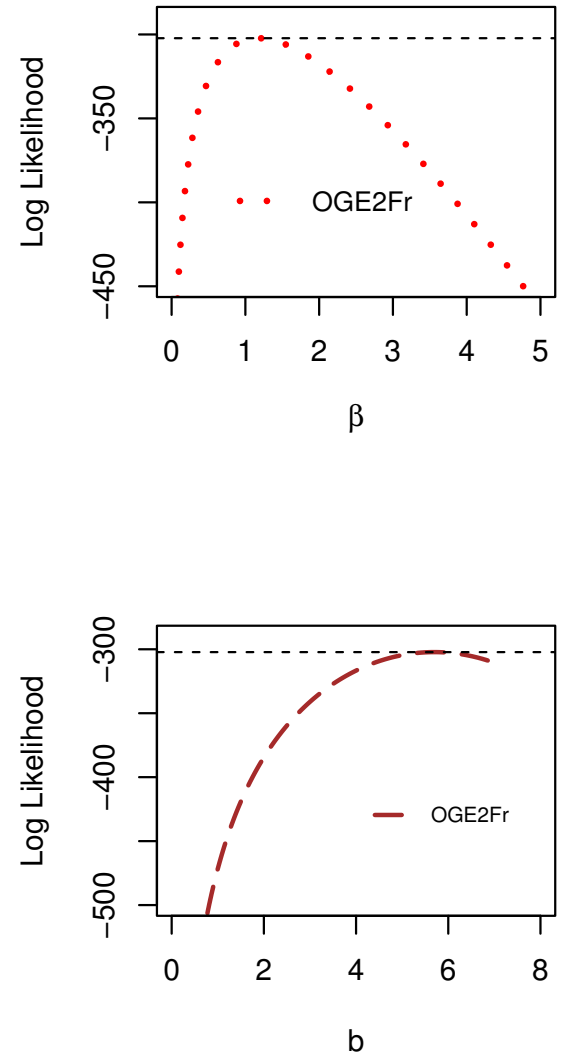

Figure 10. Profiles of the log-likelihood function for the parameters $\alpha, \beta, a$ and $b$, respectively, of the OGE2Fr for the PD1.

\subsection{Actuarial Measures}

One of the most important duties of actuarial sciences organizations is to assess market risk in a portfolio of instruments, which originates from changes in underlying factors such as equities prices, interest rates, or currency rates.One of the most important duties of actuarial sciences organizations is to assess market risk in a portfolio of instruments, which originates from changes in underlying factors such as equities prices, interest rates, or currency rates. We compute several important risk measures for the suggested distribution in this section, such as Value at Risk (VaR) and Expected Shortfall (ES), which are important in portfolio optimization under uncertainty. 


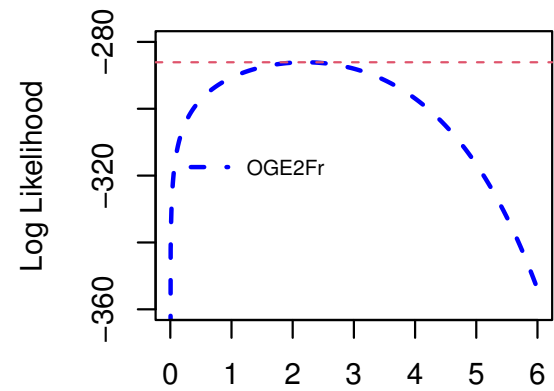

$\alpha$

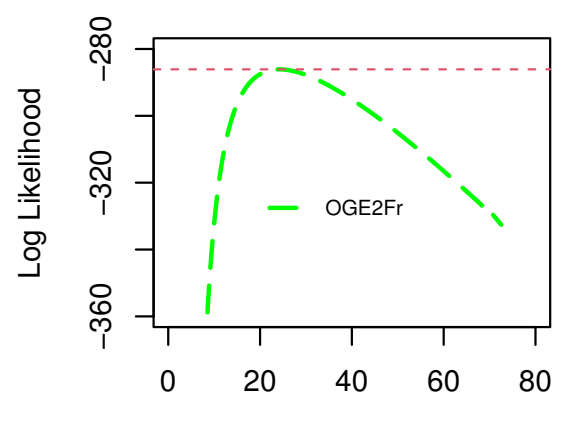

a
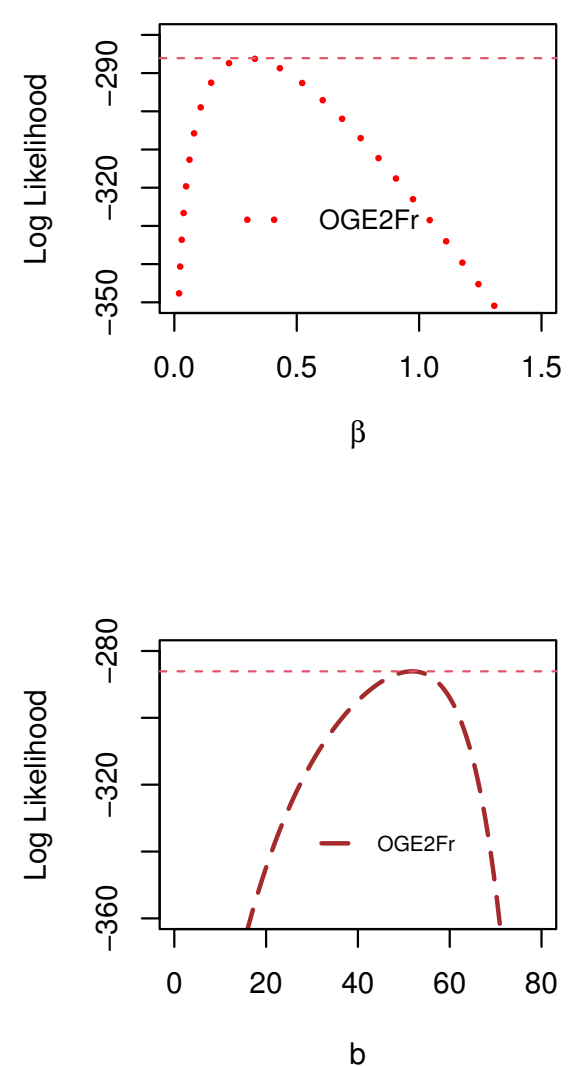

Figure 11. Profiles of the log-likelihood function for the parameters $\alpha, \beta, a$ and $b$, respectively, of the OGE2Fr for the PD2.

\subsubsection{Value at Risk}

The quantile premium principal of the distribution of aggregate losses, commonly known as Value at risk (VaR), is the most widely used measure to evaluate exposure to risk in finance. VaR of a rv is the $p$ th quantile of its cdf. If $X \sim$ OGE2Fr denotes a random variable with cdf (17), then its VaR is

$$
\operatorname{VaR}_{p}=\left[a\left\{-\log \left(1-\left[1-\log \left(1-p^{1 / \beta}\right)\right]^{-1 / \alpha}\right)\right\}\right]^{-1 / b} .
$$

\subsubsection{Expected Shortfall}

Artzner et al. $[47,48]$ recommended the use of conditional VaR instead of VaR, famously called Expected Shortfall (ES). The ES is a metric that quantifies the average loss in situations where the VaR level is exceeded. It is defined by the following expression

$$
E S_{p}=\frac{1}{p} \int_{0}^{p} V a R_{x} d x, \quad \text { where } \quad 0<p<1 .
$$

The ES of OGE2Fr is given by

$$
E S_{p}=\frac{1}{p} \int_{0}^{p}\left[a\left\{-\log \left(1-\left[1-\log \left(1-p^{1 / \beta}\right)\right]^{-1 / \alpha}\right)\right\}\right]^{-1 / b} d x
$$

Figure 12 illustrates VaR and ES for some random parameter combinations of OGE2Fr.

\subsubsection{Numerical Calculation of VaR and ES}

The results of OGE2Fr presented in Section 4 allowed us to further explore its application to these risk measures. From Table 5, we take the values of MLEs of PD1 and PD2, respectively, to measure the volatility associated with these measures. Higher values 
of these risk measures signify heavier tails while lower values indicate a much lighter tail behavior of the model. It is worth mentioning that the OGE2Fr model produced substantially more significant results than its counterparts, indicating that the model has a heavier tail. In Table 7, we show the numerical results of VaRs and ESs of PD1 and PD2, respectively, of the proposed model. For the convenience of the reader, Figure 13 show the results graphically.

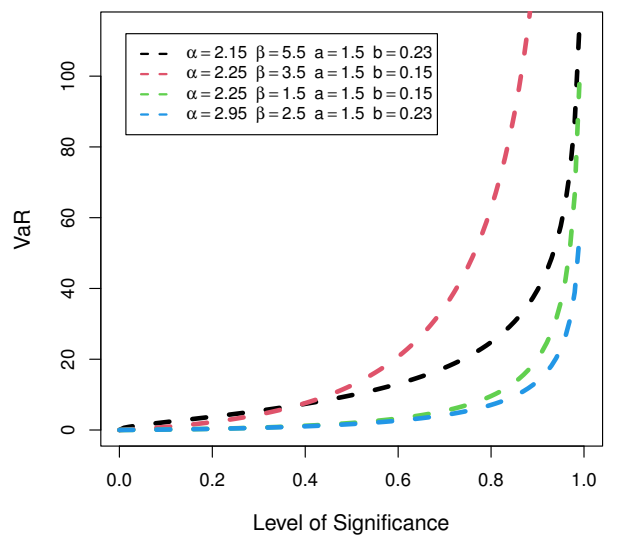

(a)

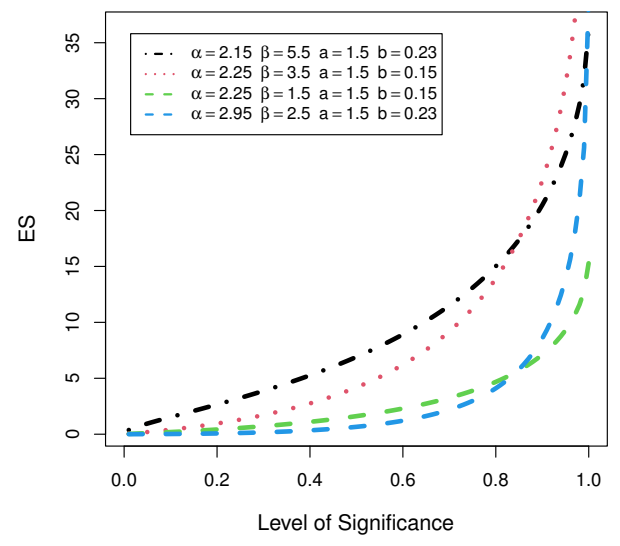

(b)

Figure 12. Plots of (a) VaR (b) ES for some parameter values.

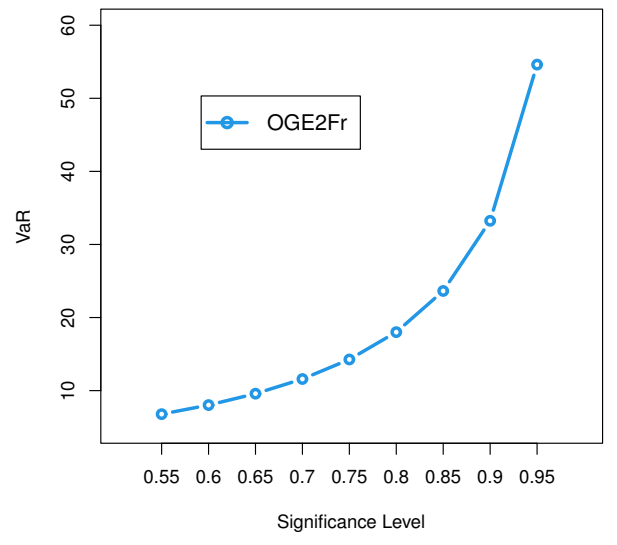

(a)

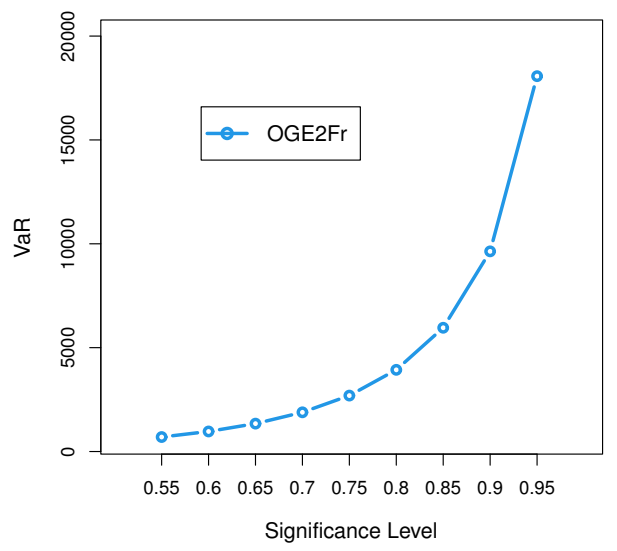

(c)

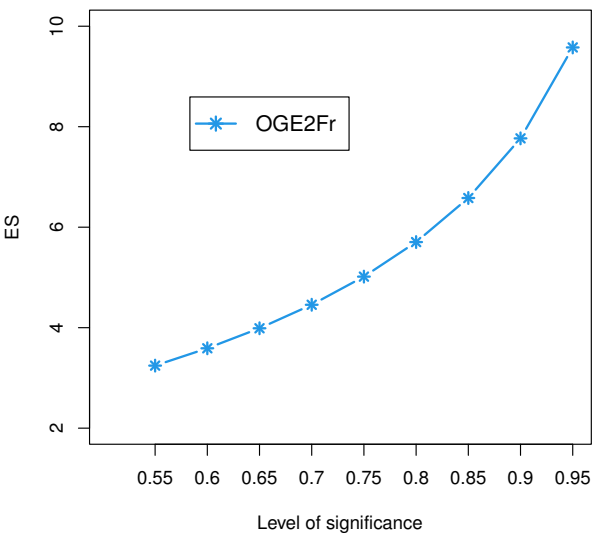

(b)

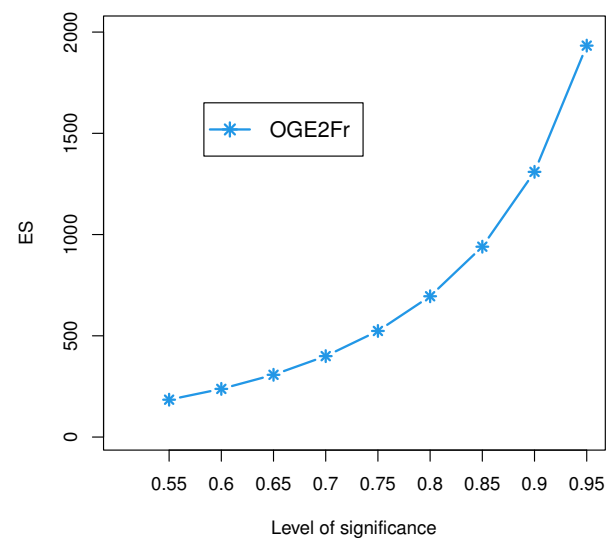

(d)

Figure 13. Estimated $\operatorname{VaR}(\mathbf{a}, \mathbf{c})$ with $\mathrm{ES}(\mathbf{b}, \mathbf{d})$ for PD1 \& PD2. 
Table 7. Numerical measures of VaRs and ESs of PD1 \& PD2.

\begin{tabular}{ccccc}
\hline Level of Significance & VaRs(PD1) & ESs(PD1) & VaRs(PD2) & ESs(PD2) \\
\hline 0.55 & 6.782 & 3.245 & 698.117 & 184.508 \\
0.60 & 8.023 & 3.589 & 965.385 & 237.819 \\
0.65 & 9.581 & 3.988 & 1343.206 & 307.461 \\
0.70 & 11.588 & 4.456 & 1887.655 & 399.670 \\
0.75 & 14.263 & 5.016 & 2693.364 & 523.906 \\
0.80 & 18.005 & 5.704 & 3932.723 & 695.305 \\
0.85 & 23.638 & 6.581 & 5957.838 & 1309.704 \\
0.90 & 33.238 & 7.768 & 9636.239 & 1933.041 \\
0.95 & 54.610 & 9.578 & $18,069.512$ & 3659.730 \\
\hline
\end{tabular}

\section{Discussion}

The OGE2-G class of distribution is proposed and studied with some mathematical properties such as ordinary and incomplete moments, mean deviations and generating functions. The maximum likelihood approach is used to estimate the model parameters. Then, we focussed our attention to one of the special member of the family defined with the Fréchet distribution, called the OGE2Fr distribution. We established the optimized maximum likelihood methodology in particular, with the goal of effectively estimating model parameters and validated their convergence by a simulation study, ensuring that the projections have asymptotic properties. To demonstrate the potentiality of the proposed model, two applications to real data sets are provided. The creation of various regression models, Bayesian parameter estimates, and studies of new data sets will all be part of a future effort. We feel that the OGE2-G family can be useful for professionals in statistical analyses beyond the scope of this research because of its several other features.

Author Contributions: Conceptualization: S.K. and M.H.T.; Methodology: W.A.; Software: O.S.B.; Validation: M.H.T., W.A. and A.A.A.; Formal analysis: S.K.; Investigation: S.K.; Resources: M.H.T.; Data curation: O.S.B. and W.A.; Writing-original draft preparation:, S.K.; Writing-review and editing: S.K.; Visualization: S.K.; Supervision: A.A.A.; Project administration: O.S.B. All authors have read and agreed to the published version of the manuscript.

Funding: This manuscript is supported by Digiteknologian TKI-ymparisto project A74338(ERDF, 357 Regional Council of Pohjois-Savo.

Data Availability Statement: https:/ / data.world/datasets/insurance/ (accesed on 28 June 2021).

Acknowledgments: The authors would like to thank the reviewers for their thoughtful remarks and recommendations, which considerably enhanced the paper's presentation.

Conflicts of Interest: The authors state that they have no conflicting interests to declare in this work.

\section{Abbreviations}

The following abbreviations are used in this manuscript:

$\begin{array}{ll}\text { GE } & \text { generalized exponential } \\ \text { LA-I } & \text { Lehmann alternatives I } \\ \text { LA-II } & \text { Lehmann alternatives II } \\ \text { OGE-G } & \text { Odd generalized exponential } \\ \text { OGE1-G } & \text { generalized odd generalized exponentia distributionl } \\ \text { OGE2-G } & \text { generalized Odd generalized exponential distribution } \\ \text { sf } & \text { survival function } \\ \text { hrf } & \text { hazard rate function } \\ \text { pdf } & \text { probability density function } \\ \text { cdf } & \text { cumulative distribution function } \\ \text { rv } & \text { random variable } \\ \text { OGE2Fr } & \text { generalized Odd generalized exponential distribution } \\ \text { Fr } & \text { Fréchet }\end{array}$




\section{References}

1. Marshall, A.W.; Olkin, I. A new method for adding parameters to a family of distributions with application to the exponential and Weibull families. Biometrika 1997, 10, 641-652. [CrossRef]

2. Gleaton, J.U.; Lynch, J.D. Properties of generalized log-logistic families of lifetime distributions. J. Probab. Stat. Sci. 2006, 4, 51-64.

3. Cooray, K. Generalization of the Weibull distribution: The odd Weibull family. Stat. Model. Int. J. 2006, 6, 265-277. [CrossRef]

4. Bourguignon, M.; Silva, R.B.; Cordeiro, G.M. The Weibull-G family of probability distributions. J. Data Sci. 2014, 12, 53-68. [CrossRef]

5. Alzatreh, A.; Famoye, F.; Lee, C. A new method for generating families of continuous distributions. Metron 2013, 71, 63-79. [CrossRef]

6. Ahsan ul Haq, M.; Elgarhy, M. The Odd Fréchet-G family of Probability distributions. J. Stat. Appl. Probab. 2017, 7, 185-201. [CrossRef]

7. Alizadeh, M.; Cordeiro, G.M.; Nascimento, A.D.C. Odd-Burr generalized family of distributions with some applications. J. Stat. Comput. Simul. 2016, 87, 367-389. [CrossRef]

8. Afify, A.Z.; Altun, E.; Alizadeh, M.; Ozel, G.; Hamedani, G.G. The Odd Exponentiated Half-Logistic-G Family: Properties, Characterizations and Applications. Chil. J. Stat. 2017, 8, 65-91.

9. Bakouch, H.; Chesneau, C.; Khan, M.N. The Extended Odd Family of Probability Distributions with Practice to a Submodel. Filomat 2018, 33, 3855-3867. [CrossRef]

10. Cordeiro, G.M.; Yousof, H.M.; Ramires, T.G.; Ortega, E.M.M. The Burr XII System of densities: Properties, regression model and applications. J. Stat. Comput. Simul. 2017, 87, 1-25. [CrossRef]

11. Chesneau, C.; Toufik, E.A. Modified Odd Weibull Family of Distributions: Properties and Applications. J. Indian Soc. Probab. Stat. 2019, 21, 259-286. [CrossRef]

12. El-Morshedy, M.; Eliwa, M.S. The odd flexible Weibull-H family of distributions: Properties and estimation with applications to complete and upper record data. Filomat 2019, 33, 2635-2652. [CrossRef]

13. Haghbin, H.; Ozel, G.; Alizadeh, M.; Hamedani, G.G. A new generalized odd log-logistic family of distributions. Commun. Stat. Theory Methods 2016, 46, 9897-9920. [CrossRef]

14. Jamal, F.; Nasir, M.; Tahir, M.H.; Montazeri, N. The odd Burr-III family of distributions. J. Stat. Appl. Probab. 2017, 6, 105-122. [CrossRef]

15. Torabi, H.; Montazari, N.H. The gamma-uniform distribution and its application. Kybernetika 2012, 48, 16-30.

16. Elmorshedy, M.; Eliwa, M.S.; Afify, A.Z. The Odd Chen Generator of Distributions: Properties and Estimation Methods with Applications in Medicine and Engineering. J. Natl. Sci. Found. Sri Lanka 2020, 48, 113-130.

17. Almamy, J.A.; Ibrahim, M.; Eliwa, M.S.; Al-mualim, S.; Yousof, H.M. The Two-Parameter Odd Lindley Weibull Lifetime Model with Properties and Applications. Int. J. Stat. Probab. 2018, 7, 57. [CrossRef]

18. Nasiru, S. Extended Odd Fréchet-G Family of Distributions. J. Probab. Stat. 2018, 2018, 2931326. [CrossRef]

19. Afify, A.Z.; Cordiero, G.M.; Mead, M.E.; Alizadeh, M.; Al-Mofleh, H.; Nofal, Z.M. The Generalized Odd Lindley-G Family: Properties and Applications. Ann. Braz. Acad. Sci. 2019, 91, e20180040. [CrossRef]

20. Korkamaz, M.C. A new family of the continuous distributions: The extended Weibull-G family. Commun. Fac. Sci. Univ. Ank. Ser. Math. Stat. 2018, 68, 248-70. [CrossRef]

21. Alex, er C.; Cordiero, G.M.; Ortega, E.M.M.; Sarabia, J.M. Generalized beta-generated distributions. Comput. Stat. Data Anal. 2012, 56, 1880-1897. [CrossRef]

22. Korkamaz, M.C.; Genc, A.I. A new generalized gwo-sided class of distributions with an emphasis on two-sided generalized normal distribution. Commun. Stat.-Simul. Comput. 2015, 46, 1441-1460. [CrossRef]

23. Korkamaz, M.C.; Cordiero, G.M.; Yousof, H.M.; Pescim, R.R.; Afify, A.Z.; Nadarajah, S. The Weibull Marshall-Olkin family: Regression model and application to censored data. Commun. Stat.-Theory Methods 2018, 48, 4171-4194. [CrossRef]

24. Korkamaz, M.C.; Yousof, H.M.; Hamedani, G.G. The Exponential Lindley Odd Log-Logistic-G Family: Properties, Characterizations and Applications. J. Stat. Theory Appl. 2018, 17, 554-571. [CrossRef]

25. Korkamaz, M.C.; Altun, E.; Yousof, H.M.; Afify, A.Z.; Nadarajah, S. The Burr-X Pareto Distribution: Properties, Applications and VaR Estimation. J. Risk Financ. Manag. 2017, 11, 1. [CrossRef]

26. Korkamaz, M.C.; Alizadeh, M.; Yousof, H.M.; Butt, N.S. The Generalized Odd Weibull Generated Family of Distributions: Statistical Properties and Applications. Pak. J. Stat. Oper. Res. 2018, 542, 541-556. [CrossRef]

27. Korkamaz, M.C.; Yousof, H.M.; Alizadeh, M.; Hamedani, G.G. The Topp-Leone Generalized Odd Log-Logistic Family of Distributions: Properties, Characterizations and Applications. Commun. Fac. Sci. Univ. Ank. Ser. A1 Math. Stat. 2018, 68, $1506-1527$. [CrossRef]

28. Alizadeh, M.; Korkamaz, M.C.; Almanay, J.A.; Afify, A.Z. Another odd log-logistic logarithmic class of continuous distributions. J. Stat. Stat. Actuar. Sci. 2018, 2, 55-72.

29. Silva, F.G.; Ramos, M.W.; Percontini, A.; Venâncio, R.; De Brito, E.; Cordeiro, G.M. The Odd Lindley-G Family of Distributions. Austrian J. Stat. 2017, 35, 281-308. [CrossRef]

30. Korkamaz, M.C.; Yousof, H.M.; Ali, M.M. Some Theoretical and Computational Aspects of the Odd Lindley Fréchet Distribution. J. Stat. Stat. Actuar. Sci. 2017, 2, 129-140. 
31. Korkamaz, M.C.; Altun, E.; Yousof, H.M.; Hamedani, G.G. The Hjorth's IDB generator of distributions: Properties, characterizations, regression modelling and applications. J. Stat. Theory Appl. 2020, 19, 59-74. [CrossRef]

32. Tahir, M.H.; Cordeiro, G.M.; Alizadeh, M.; Mansoor, M.; Zubair, M.; Hamedani, G.G. The odd generalized exponential family of distributions with applications. J. Stat. Distrib. Appl. 2015, 2, 1. [CrossRef]

33. Morad, A.; Ghosh, I.; Yusuf, H.M.; Rasekhi, M.; Hamedani, G.G. The generalized odd generalized exponential family of distributions. J. Data Sci. 2017, 15, 443-465. [CrossRef]

34. Tahir, M.H.; Nadarajah, S. Parameter induction in continuous univariate distributions: Well-established G families. $A A B C$ 2015, 87 , 539-568. [CrossRef]

35. Aarset, M.V. How to identify bathtub hazard rate. IEEE 1987, 36, 106-108. [CrossRef]

36. Rényi, A. On measures of entropy and information. In Proceedings of the 4th Berkeley Symposium on Mathematical Statistics and Probability, Berkeley, CA, USA, 20-30 July 1960; Volume 1, pp. 547-561.

37. Shaked, M.; Shantikumar, J.G. Stochastic Orders and Their Applications; Springer: New York, NY, USA, 1994.

38. Nadarajah, S.; Gupta, A.K. The Beta Fréchet distribution. Far East J. Theor. Stat. 2004, 14, 15-24.

39. Souza, W.B.; Cordeiro, G.M.; Simas, A.B. Some Results for Beta Fréchet Distribution. Commun. Stat.-Theory Methods 2011, 40, 798-811. [CrossRef]

40. Cordeiro, G.M.; De-Castro, M. A new family of generalized distributions. J. Stat. Comput. Simul. 2011, 81, 883-898. [CrossRef]

41. Cordeiro, G.M.; Ortega, E.M.M.; Cunha, D.C.C. The exponentiated generalized class of distributions. J. Data Sci. $2013,11,1-27$. [CrossRef]

42. Krishna, E.; Jose, K.K.; Ristic, M.M. Application of Marshall-olkin Fréchet distribution. Commun. Stat.-Simul. Comput. 2013, 42, 76-89. [CrossRef]

43. Nadarajah, S.; Kotz, S. The exponentiated Fréchet distribution. Interstat Electron. J. 2003, 92, 97-111.

44. Da Silva, R.V.; De Andrade, T.A.N.; Maciel, D.B.M.; Campos, R.P.S.; Cordeiro, G.M. A New Lifetime Model: The Gamma Extended Frechet Distribution. J. Stat. Theory Appl. 2013, 12, 39-54. [CrossRef]

45. Abbas, S.; Taqi, S.A.; Mustafa, F.; Murtaza, M.; Shahbaz, M.Q. Topp-Leone Inverse Weibull Distribution: Theory and Application. Eur. J. Pure Appl. Math. 2017, 10, 1005-1022.

46. Fréchet, M. Sur la loi de probabilite de lecart maximum. APM 1927, 6, 110-116.

47. Artzner, P.; Delbaen, F.; Eber, J.M.; Heath, D. Thinking Coherently. Risk 1997, 10, 68-71.

48. Artzner, P. Application of coherent risk measures to capital requirements in insurance. NAAJ 1999, 3, 11-25. [CrossRef] 